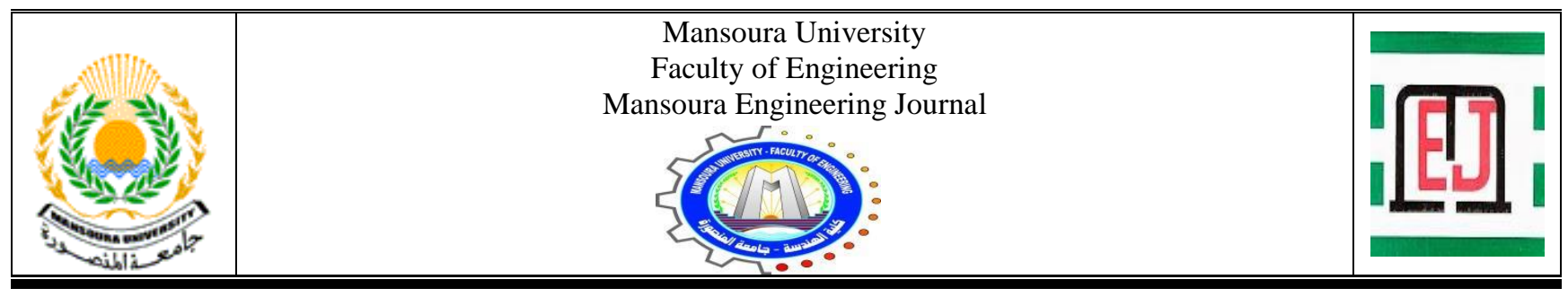

\title{
Effect of Solar Radiation on Thermal Comfort in Inner Ambient Behind a Building Wall with Facing Material of Different Colors
}

\author{
Rasha Mazen Abdel Salam
}

\begin{tabular}{|l|}
\hline KEYWORDS: \\
Solar radiation; \\
Thermal Comfort; Wall- \\
facing $\quad$ materials; \\
Albedo; $\quad$ Reflection \\
coefficient; Absorption \\
factor; Heat protection; \\
Heat insulation \\
\end{tabular}

\begin{abstract}
This paper is aimed at investigating the effect of solar radiation on the inner ambient behind a building wall with facing material of different colors. At the wall outer-surface, the incident radiation results in a reflected radiation into the outer ambient depending on the reflection-coefficient of the wall-facing material and a transmitted radiation in the wall, whose intensity decays through its propagation in the wall depending on the absorption-factor of the wall material. At the wall inner-surface, the transmitted-radiation results in a reflected-radiation inside the wall and a penetrated-radiation into the innerambient of the wall. The sequence of radiation-propagation is continued with incidence, reflection and penetration at the wall inner-surface as well as incidence and reflection at the wall outer-surface.. To the author's knowledge, this sequence of radiation-propagation is evaluated numerically -for the first time- in the present paper. The absorbed-radiation intensity in the wall and the penetrated-radiation intensity into inner-ambient are calculated for varying albedo-values according to color of the facing-material and different values of absorption-coefficient of the wall material. The proposed method justifies that the wall-facing materials with white color realize heat-protection in summer and heat-insulation in winter with a subsequent improvement of the thermal comfort. The temperature of the inner-ambient with facing-material of darkcolor is higher than that with white-color in agreement with previous findings based on measurements for small-scale building walls by others and measurements by the author for local buildings in Assiut city, Egypt as well as predictions using computer software packages.
\end{abstract}

\section{( I I}

التطور في انماط الحياه وتكوين مجتمعات عمر انية اصبح هدف الإن الإنهاء

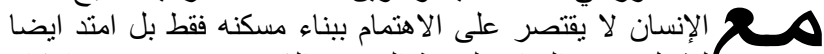

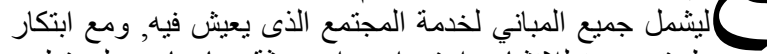

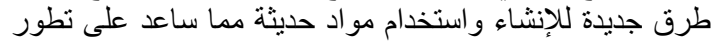

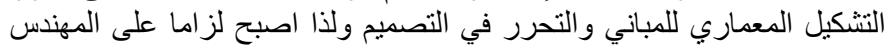

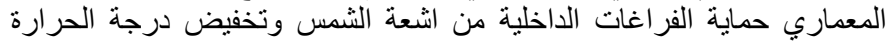

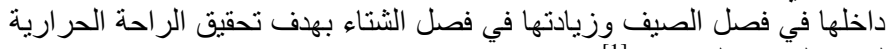

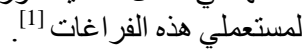

هذا وقد تم استخدام طريقة تعقب الاشعاع الثمسي لحساب معدلات الات الانتقال

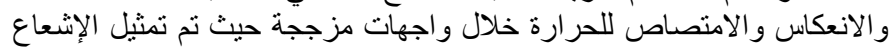

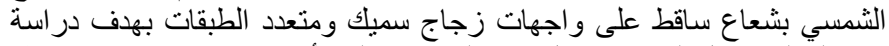

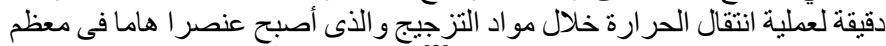

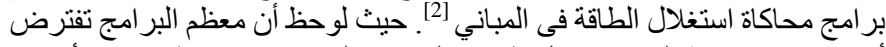

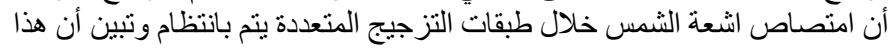

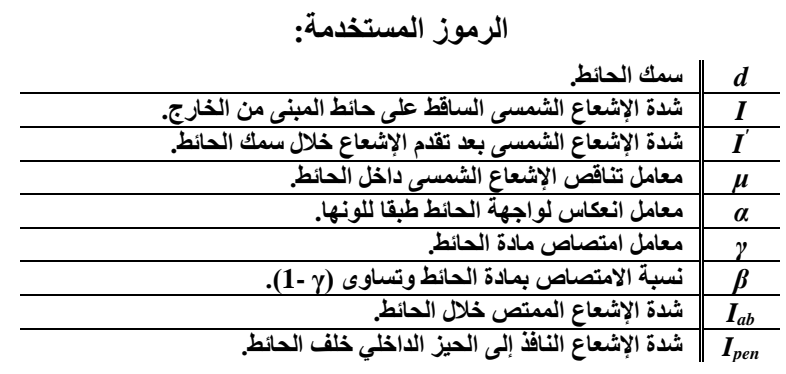

Received: (23 January, 2021) - Revised: (03 June, 2021) - Accepted: (05 June, 2021)

Corresponding Author: Rasha Mazen Abdel Salam, Assistant Professor at Faculty of Engineering, Architectural Dept., Sohag University, (e-mail: rasha_mazen@eng.sohag.edu.eg,rasha_mazen05@yahoo.com). 


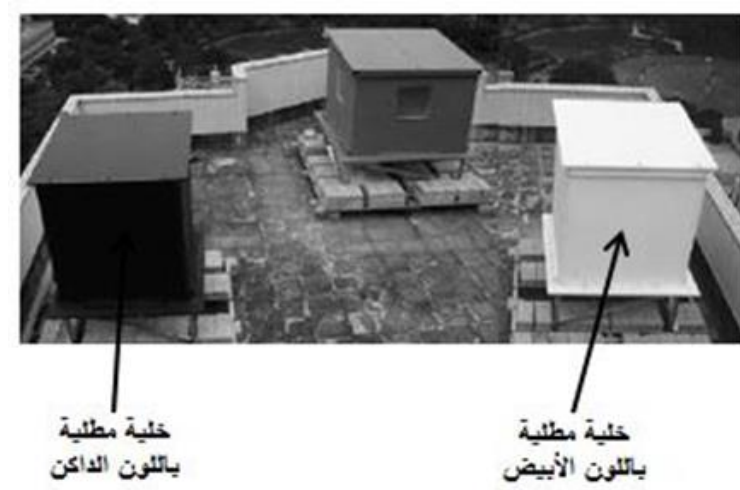

شكل (2): الخليتين الذين تم استخدامهم في القياس [5]
الفرض لم يعد ساري المفعول فى التزجيج السميك و الذى يكثر استخدامه فى المباني

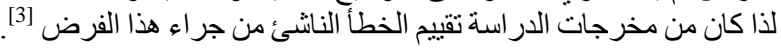

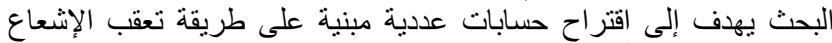

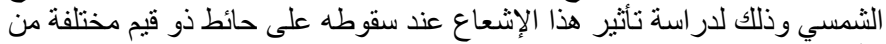

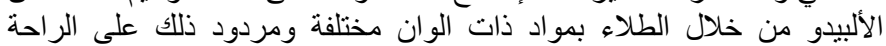

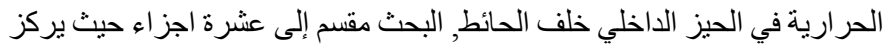

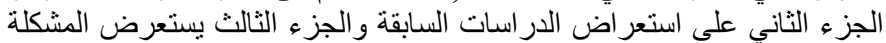

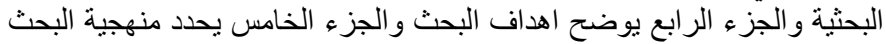

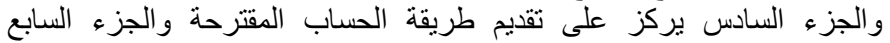

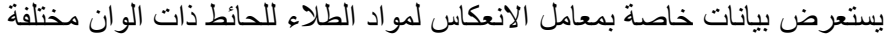

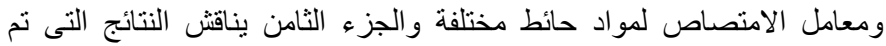

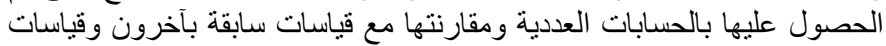

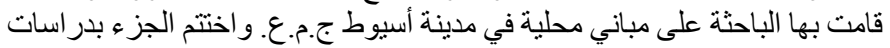

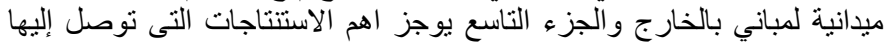

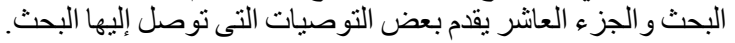

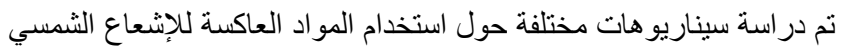

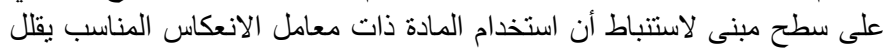

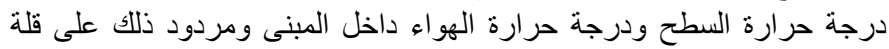

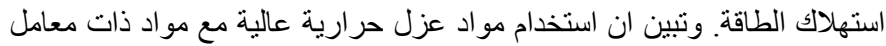

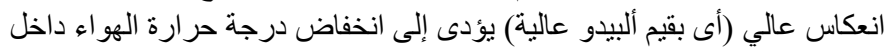

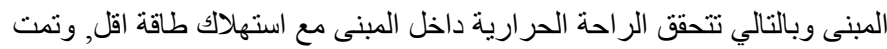

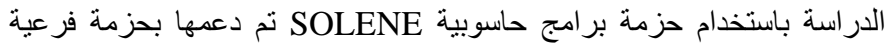
SOLENE-MICRCLIMATE تختص بالتصميم الحضري المستدام [6. تم إنشاء نموذجين خشبيين لمبنى ذو ثلاث طو ابق أبعادهم 1,2 × 1,2 × 1,8 م

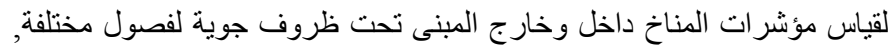

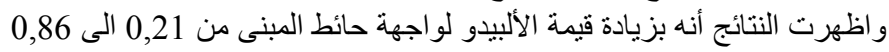

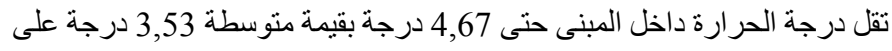

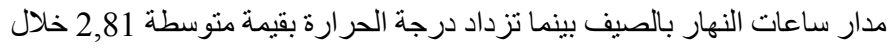

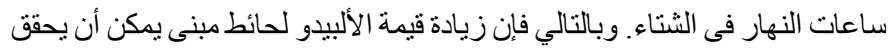

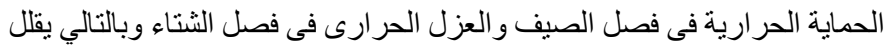

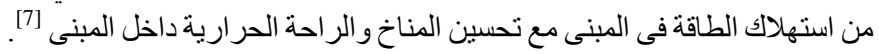

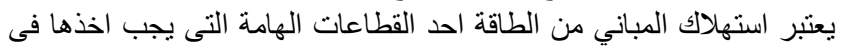

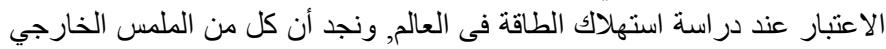

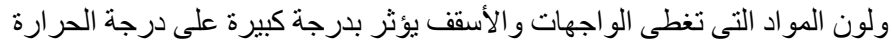

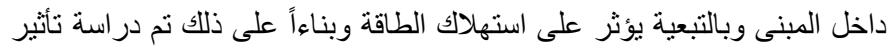

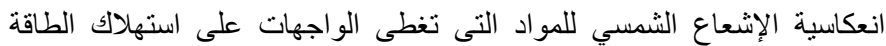

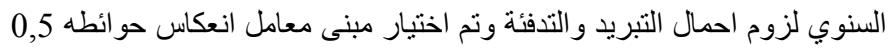
كمرجع للمقارنة مع مبنيين أحدهما معامل انعكاس حوائطه

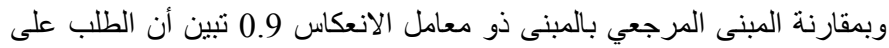

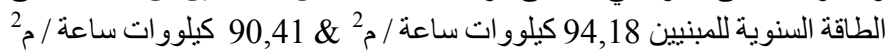

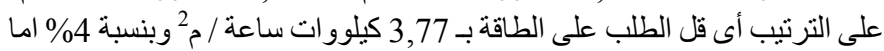

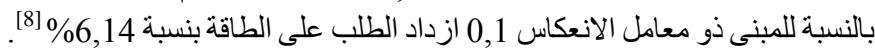

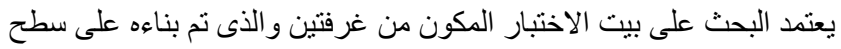

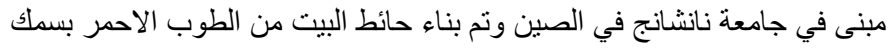

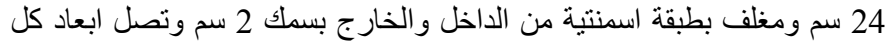

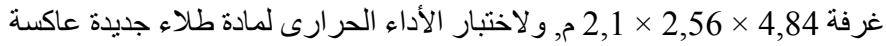

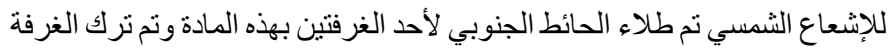

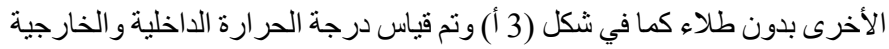

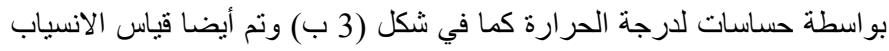

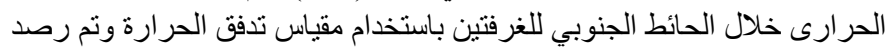

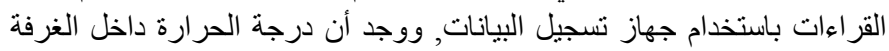

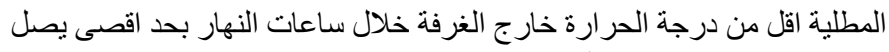

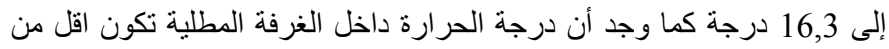

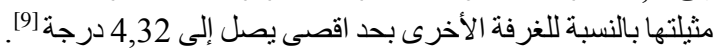

\section{II}

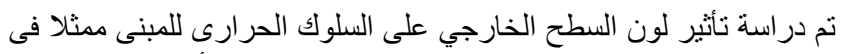

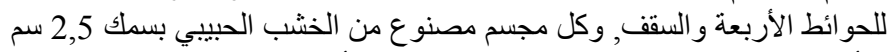

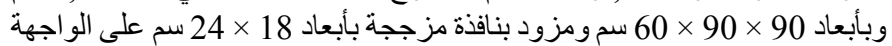
الجنوبية للمجسم وستارة على النافذة بأبعاد 22 × 30 سم وتسمح الفتاد

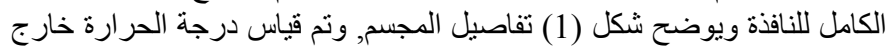

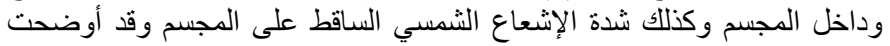

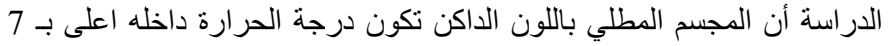

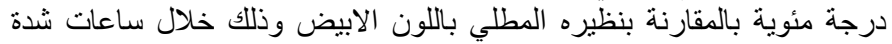
الإشعاع الثمسي القصوى مقابل فرق درجة حر ارة من بل 4 - 8 درجة مئوية للمباني الفعلية [4] لدراسة تأثير لون الغلاف الخارجي لمبنى على درجة الحرارة داخله تم بناء

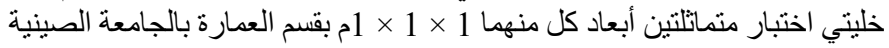
في هونج كونج وكل خلية مزودة بفتحنين في حائطين منقابلين ابعاد كل من منهما

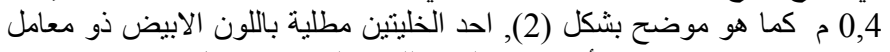

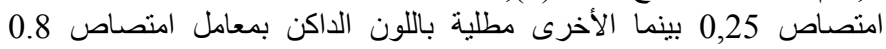

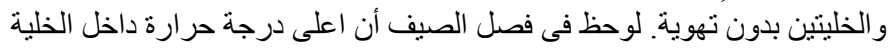

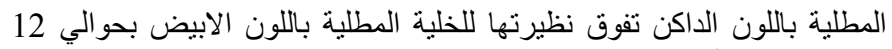

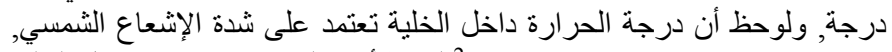

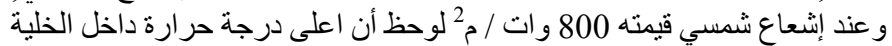

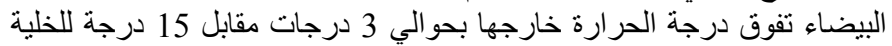

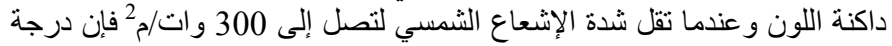

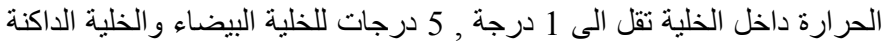

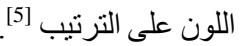

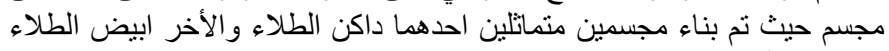

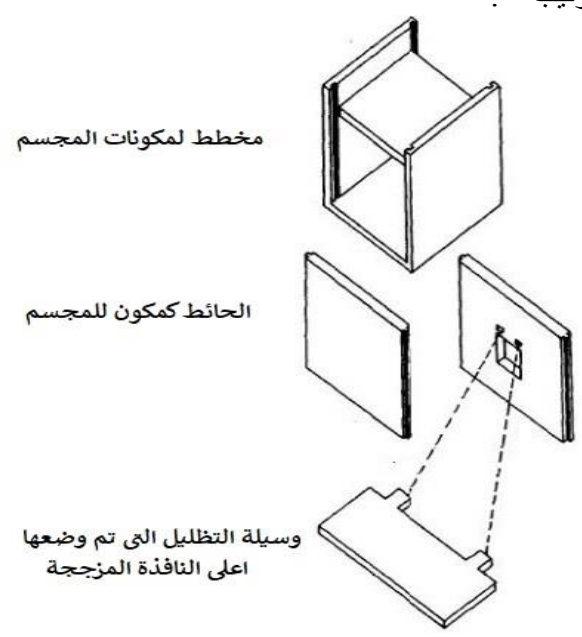

شكل (1): شكل المجسم المستخدم في القياس [4]. 

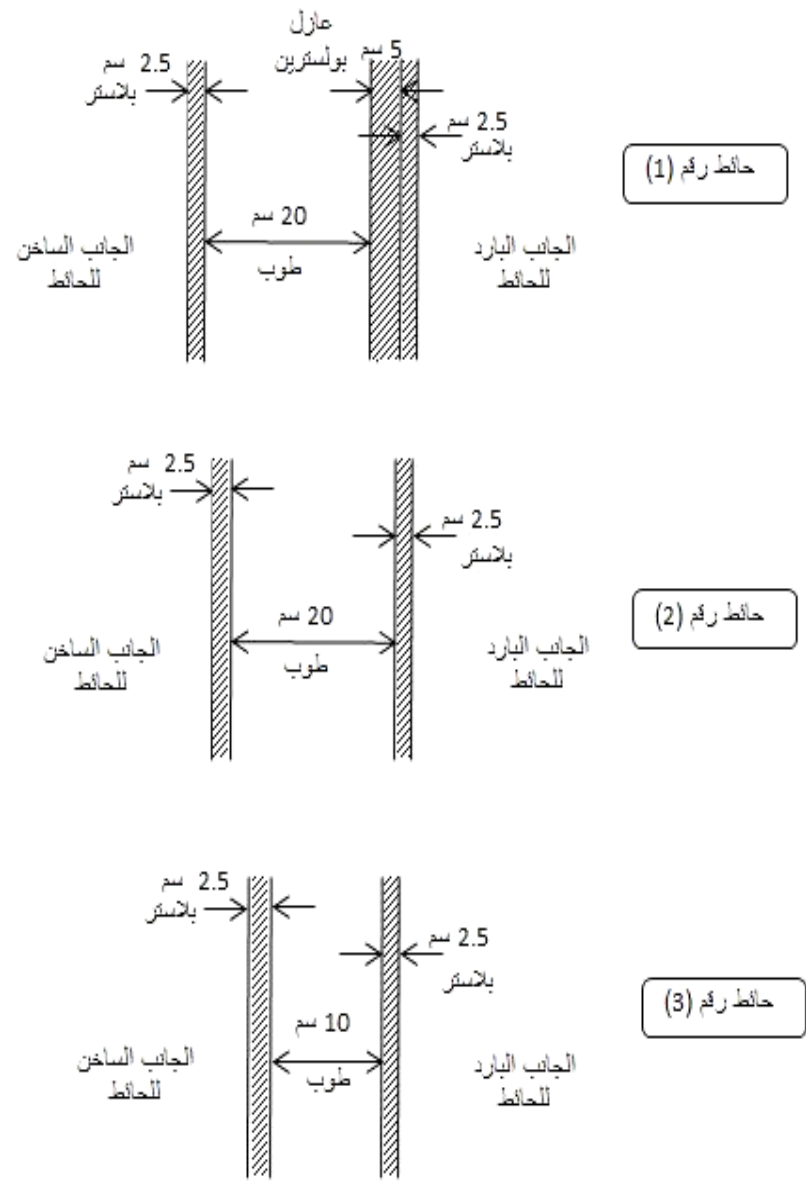

حأف زئم (3)

شكل (4) الحو ائط التى نم استخدامها في الاختبارات [12].

\section{III}

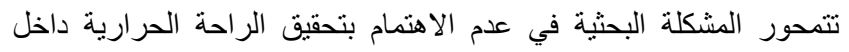

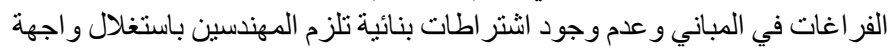

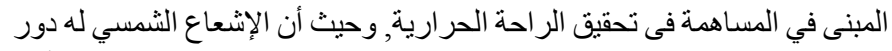

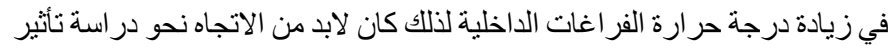

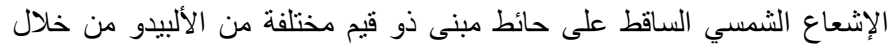

الطلاء بمو اد ذات الو ان مختلفة بهدف تحسين الر احة الحر ارية داخل الفر الفات

\section{IV}

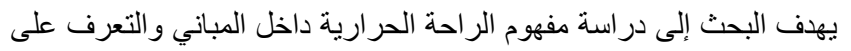

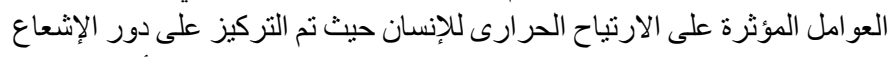

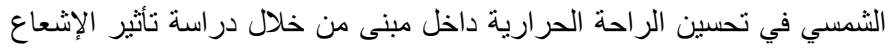

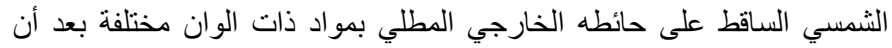

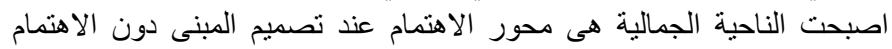
بالعو امل المناخية التى تلعب دور أساسي في تحقيق كفاءة الأداء الوظيفي لأقى مبنى.

\section{V . منهجية البحث}

لتحقيق الاهداف السابقة يتبع البحث الحالي منهجين و هما:

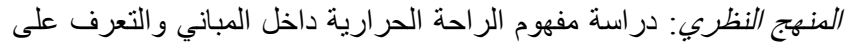

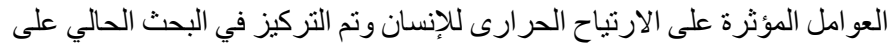

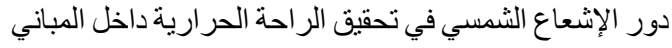

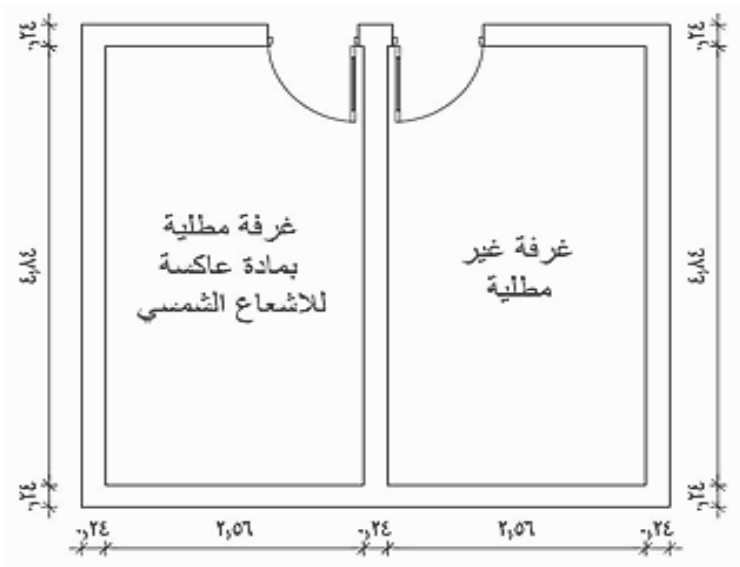

(i)

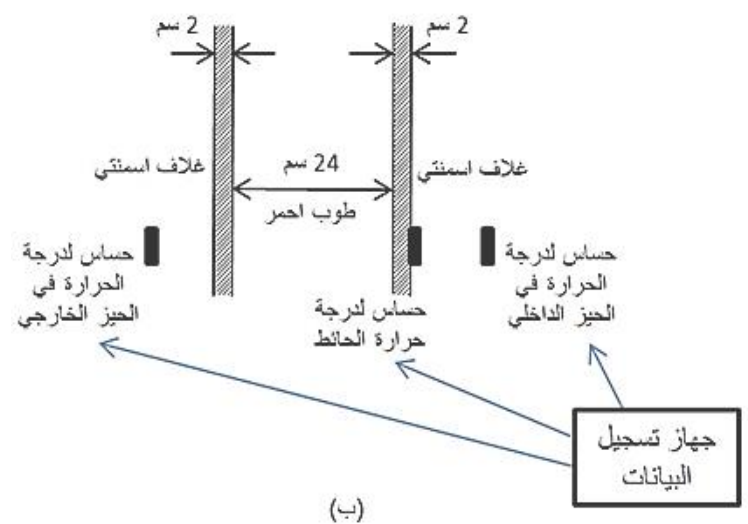

شكل (3): أ ـ مخطط لغرفتي الاختبار ب ـ مكان حساسات قياس درجة الحرارة [9].

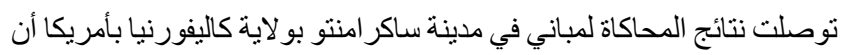

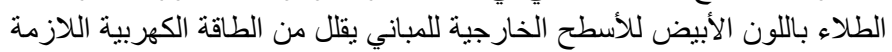

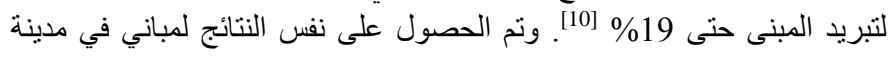

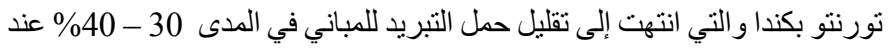

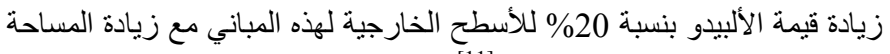

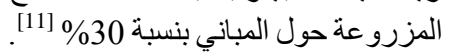

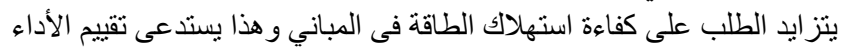

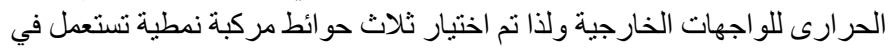
مصر للتحقق من الأداء الحرارى لهار وابعاد الحوائط 149 × × 124 سم ويختلف

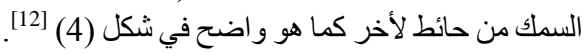

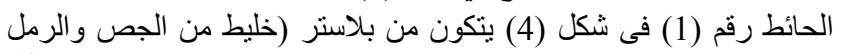

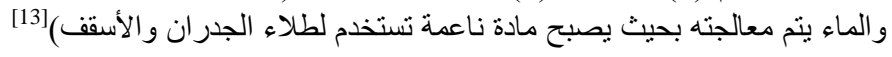

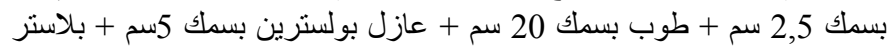

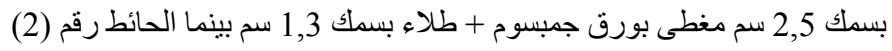

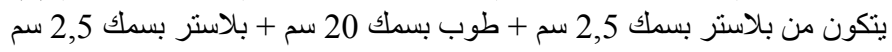
بينما الحائط رقم (3) يتكون من بلاستر بسمك 2,5 2,5 سم + طوب 10 سم سم + بلاستر

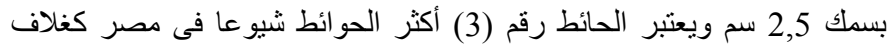

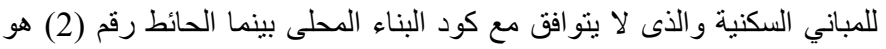

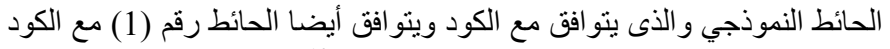

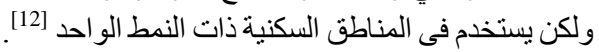

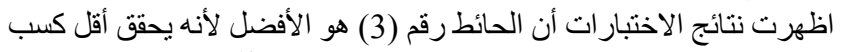

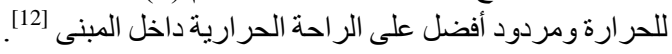




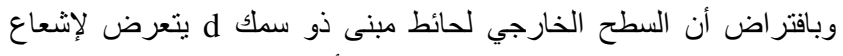

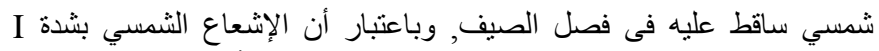

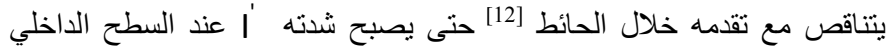

للحائط حيث:

$$
\mathrm{I}_{\mathrm{d}}=\mathrm{I} \mathrm{e}^{-\mu \mathrm{d}}=\mathrm{I} \gamma
$$

باعتبار أن ز هى معامل الامتصاص الذى يعتمد على نوع المادة المستخدمة فى

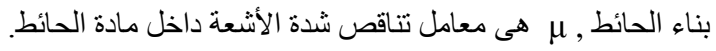

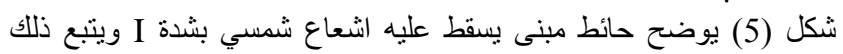

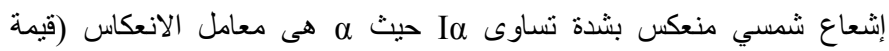

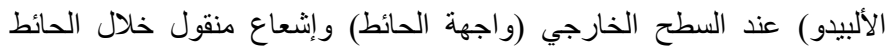

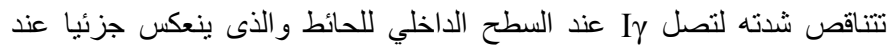

السطح الداخلي و الباقي ينفذ إلى الحيز الداخلي خلف الحئ الحائط.

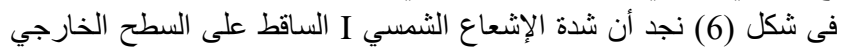

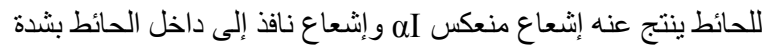

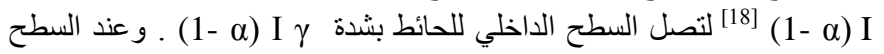

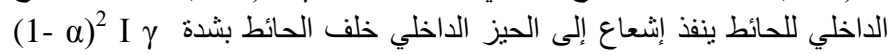

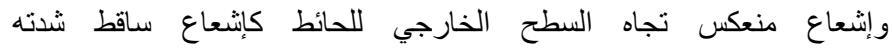

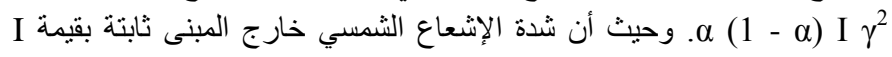

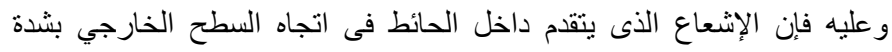

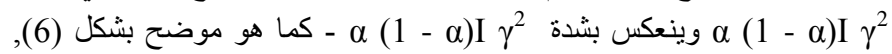
ويستمر تقدم الإشعاعات ما بين ساقط ومنعكس ونافذ على السطح الداخلي للحائط

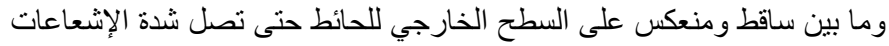
إلى قيم صغيرة جدا وبالتالي يمكن إهمالها.

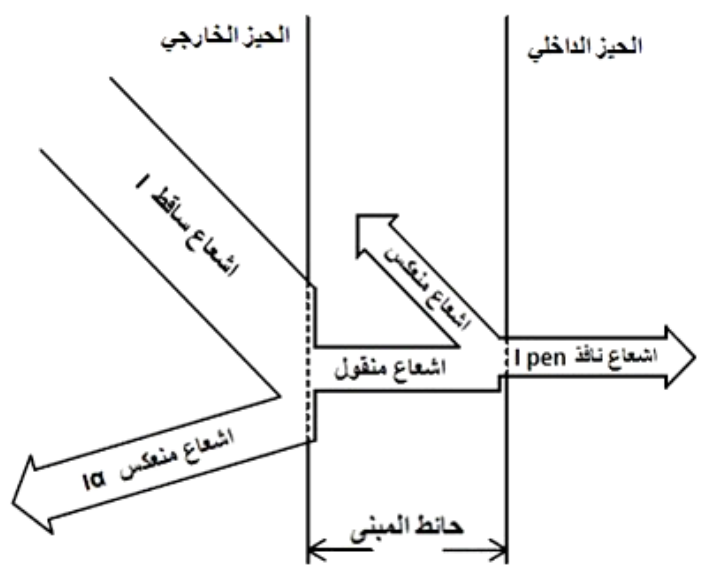

شكل (5): الإشعاعات الساقطة و المنعكسة و المنقولة و النافذة خلال حائط مبنى.

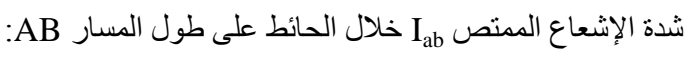
$\left(I_{\mathrm{ab}}\right)_{\mathrm{AB}}=\mathrm{I}(1-\alpha)-\mathrm{I}(1-\alpha) \gamma=\mathrm{I}(1-\alpha)(1-\gamma)$

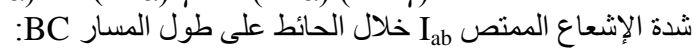
$\left(\mathrm{I}_{\mathrm{ab}}\right)_{\mathrm{BC}}=\alpha \mathrm{I}(1-\alpha) \gamma-\alpha \mathrm{I}(1-\alpha) \gamma^{2}=\alpha \mathrm{I}(1-\alpha) \gamma(1-\gamma)$

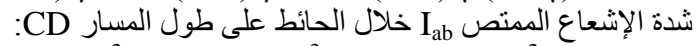
$\left(\mathrm{I}_{\mathrm{ab}}\right)_{\mathrm{CD}}=-\alpha \mathrm{I}(1-\alpha) \gamma^{2}+\alpha \mathrm{I}(1-\alpha) \gamma^{3}=-\alpha \mathrm{I}(1-\alpha) \gamma^{2}(1-\gamma)$

ويتكرر ذلك بنفس الطريقة خلال المسارين DE , EF, فيكون إجمالي شدة الإشعاع المثتص Iab خلال الحائط يعبر عنه بـ المبل:
الهنهج النطبيقي: نم اقتراح حسابات عددية مبنية على طريقة تعقب الإشعاع

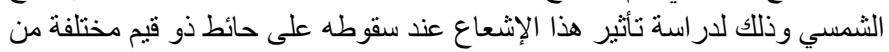

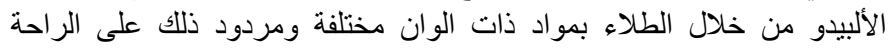

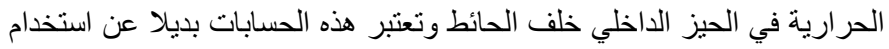

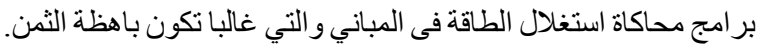

\section{VI . . هيكل البحث}

حيث أن الراحة الحرارية من العناصر التى لا يمكن أن تقاس بطريقة مباشرة

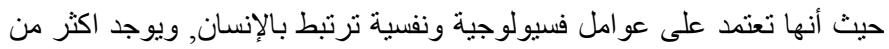

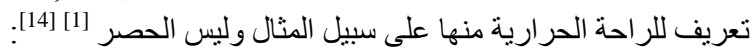

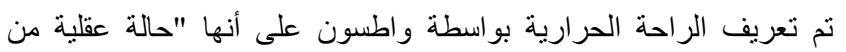

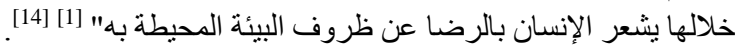

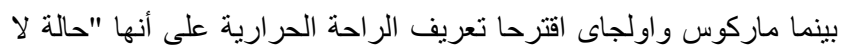

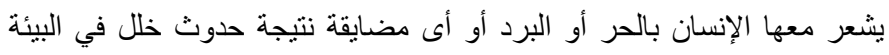

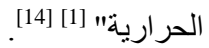

وبالتالي يمكن تعريف الراحة الحرارية على أنها "شعور الإنسان بالراحة

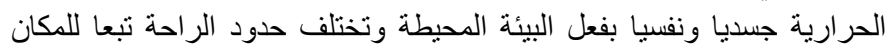

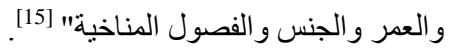

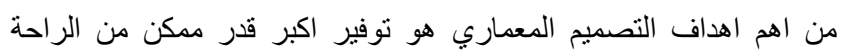

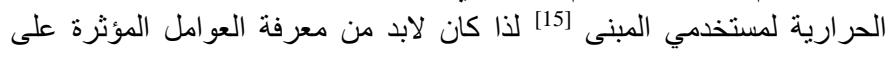

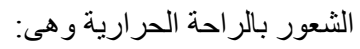

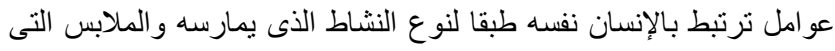

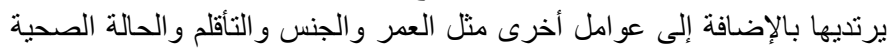
[17] [16]

عو امل ترتبط بالظروف المناخية المحيطة حيث توجد اربعة عوامل مناخية

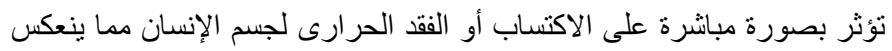

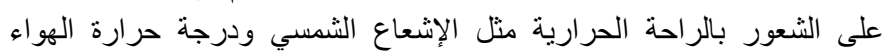

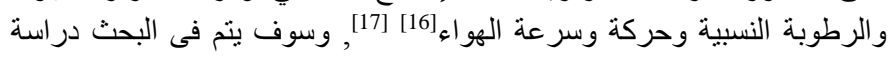

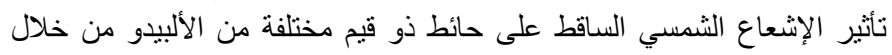

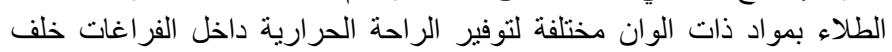
الحائط. يعتبر الاشعاع الثمسي من اكثر العوامل المؤثرة على الغلاف الخارجي

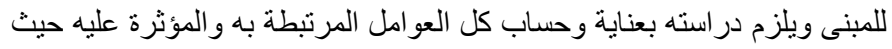

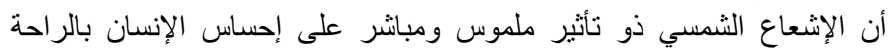

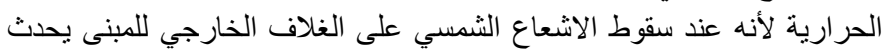

تبادل حر ارى بين الفر اغ الداخلي والخارجي عبر الحائط الخارجي للمبنى [14] الفئ.

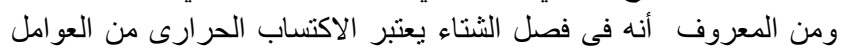

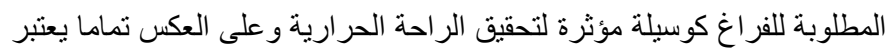

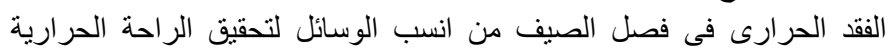

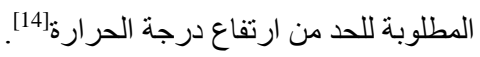

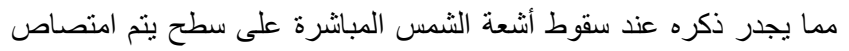

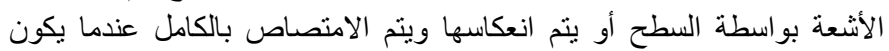

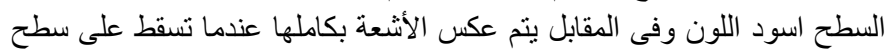

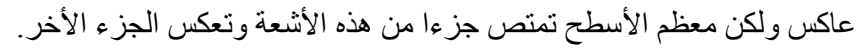

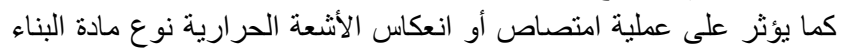

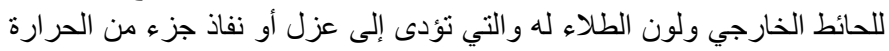

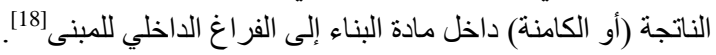




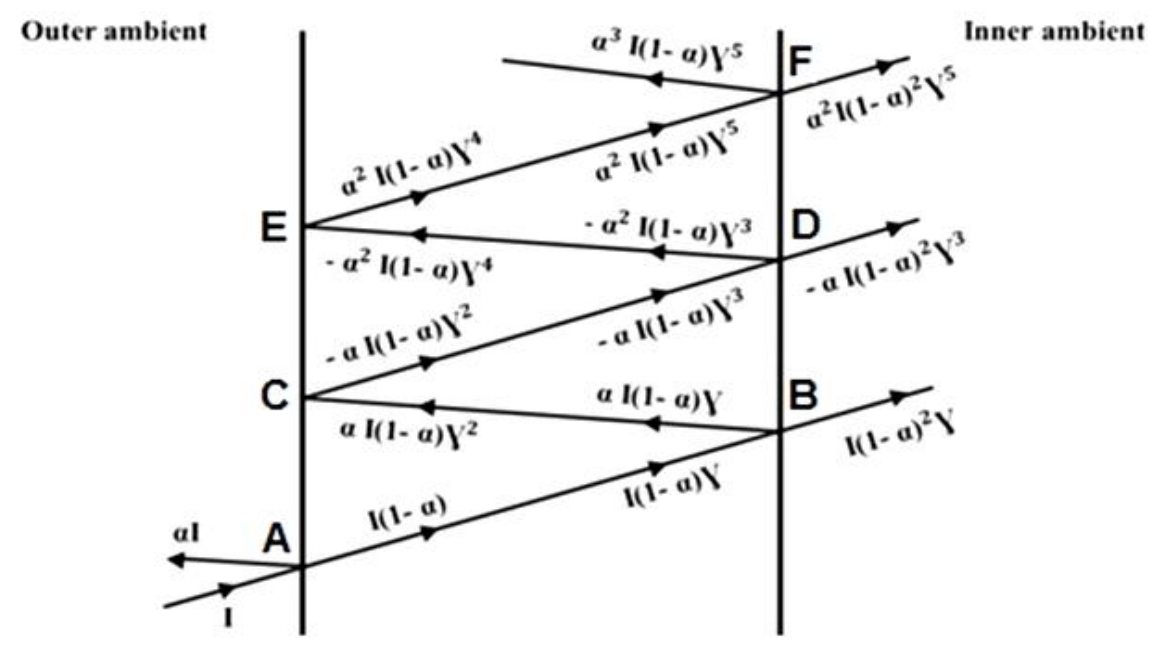

شكل (6): الانعكاسات المتعددة لحائط مطلى بمادة ذات قيمة ألبيدو عالية.

وتكون شدة الإشعاع النافـ I إلى داخل المبنى مساو لمجموع قيم شدة الأشعة النافذة عند F, D , B ويعبر عنه بـ :

$\mathrm{I}_{\mathrm{pen}}=(1-\alpha)^{2} \mathrm{I} \gamma-\alpha(1-\alpha)^{2} \mathrm{I} \gamma^{3}+\alpha^{2}(1-\alpha)^{2} \mathrm{I} \gamma^{5}+\ldots \ldots$

$$
=\mathrm{I} \gamma(1-\alpha)^{2}\left[1-\alpha \gamma^{2}+\alpha^{2} \gamma^{4}-\ldots . .\right] \text {. }
$$

\begin{tabular}{|c|c|c|c|c|c|}
\hline (d) & (c) & (B) & (A) & \multirow{2}{*}{\multicolumn{2}{|c|}{ ل لون المادة }} \\
\hline 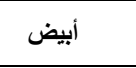 & أحمر - بنضى محمر & رمادي & 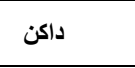 & & \\
\hline $0,90-0,70$ & $0,30-0,20$ & $0,35-0,15$ & $0,10-0,05$ & 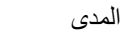 & \multirow{2}{*}{ معامل الانعكاس (قيمة الألبيدو) م } \\
\hline 0,80 & 0,25 & 0,25 & 0,075 & قيمة متوسطة & \\
\hline $0,30-0,10$ & $0,80-0,70$ & $0,75-0,65$ & $0,90>$ & 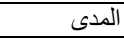 & \multirow{2}{*}{ 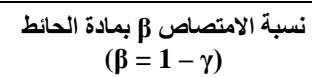 } \\
\hline 0,20 & 0,75 & 0,70 & 0,95 & قيمة متوسطة & \\
\hline
\end{tabular}

$$
\begin{aligned}
& \mathrm{I}_{\mathrm{ab}}=\left(\mathrm{I}_{\mathrm{ab}}\right)_{\mathrm{AB}}+\left(\mathrm{I}_{\mathrm{ab}}\right)_{\mathrm{BC}}+\left(\mathrm{I}_{\mathrm{ab}}\right)_{\mathrm{CD}}+\left(\mathrm{I}_{\mathrm{ab}}\right)_{\mathrm{DE}} \ldots \ldots+ \\
& \mathrm{I}_{\mathrm{ab}}=\mathrm{I}(1-\alpha)(1-\gamma)+\mathrm{I} \gamma \alpha(1-\alpha)(1-\gamma)+\left(-\alpha \mathrm{I}(1-\alpha) \gamma^{2}(1\right. \\
& -\gamma))+\ldots \ldots . . \\
& =\mathrm{I}(1-\alpha)(1-\gamma)\left[1+\gamma \alpha-\gamma^{2} \alpha-\gamma^{3} \alpha^{2}+\right.
\end{aligned}
$$

جدول (1)

قيم معاملات الانعكاس A لو اجهة حائط ذات الو ان مختلفة وكذلك قيم نسب الامتصاص B لمو اد الحائط]].

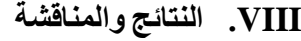

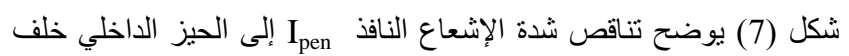

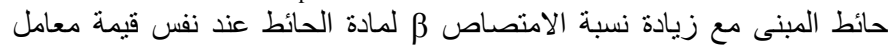

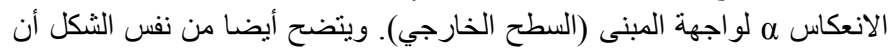

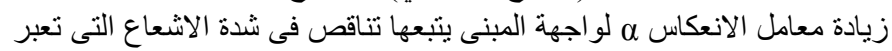

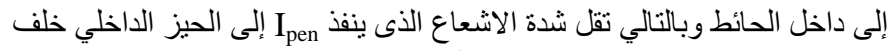

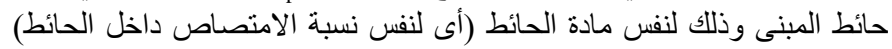

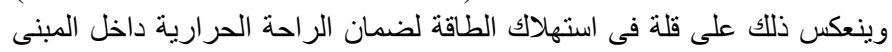

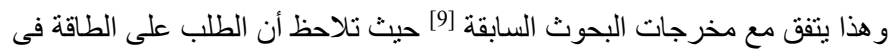

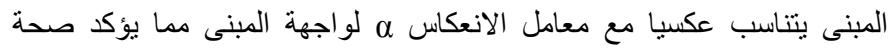
الحسابات العددية في البحثي.
تم عرض نتائج البحث في ثلاث اجزاء حيث يختص الجزء الألماء الأول بدراسة

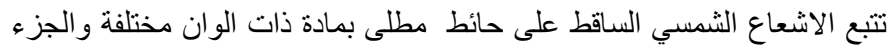

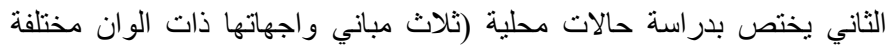

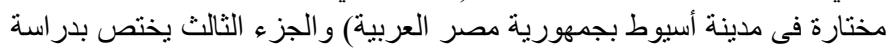

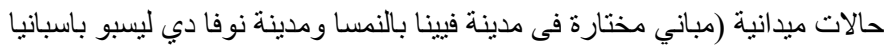
ومدينة لوس انجلوس بأمريكا).

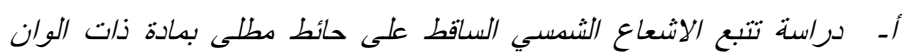

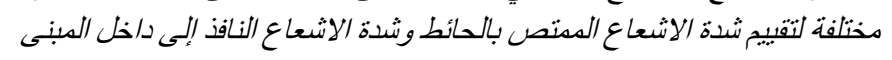

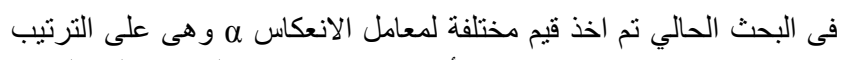
0,25 , 0,6 , 0,7 , 0,8, واعتبر أن شدة الإشعاع I الساقط على السطح الخارجي للمبنى مساوى للو احد. 


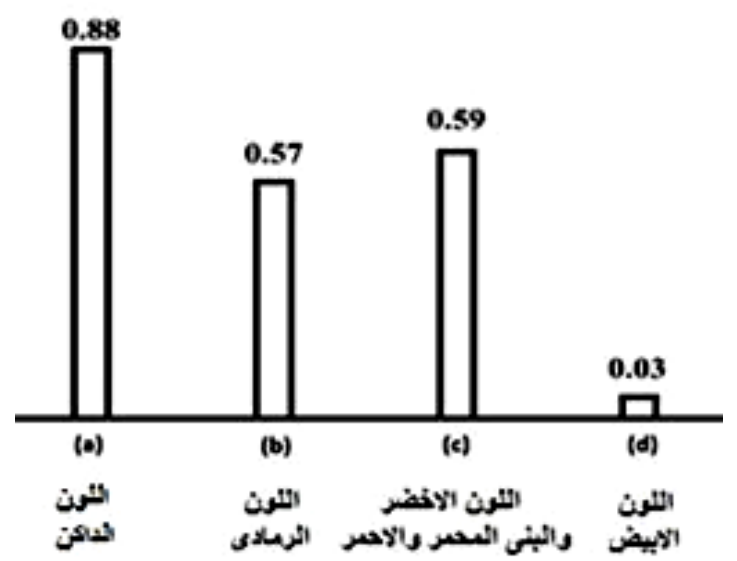

شكل (9): شدة الاشعاع الممتص Iab خلال حائط مبنى ذو مو اد ذات الو ان مختلفة.

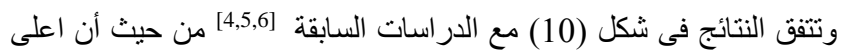

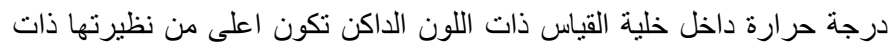

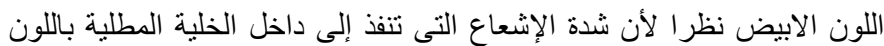

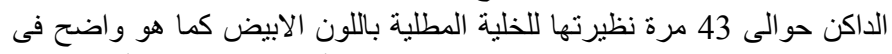

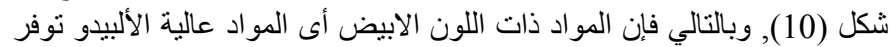

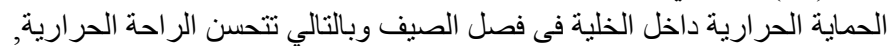
مما يؤكد صحة الحسابات العددية في البحث.

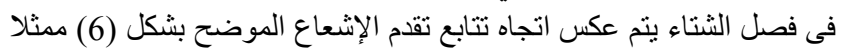

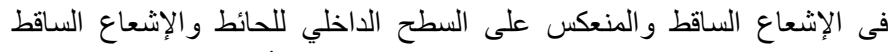

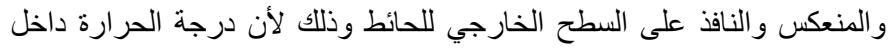

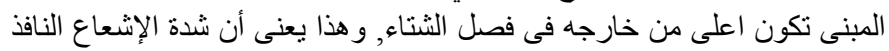

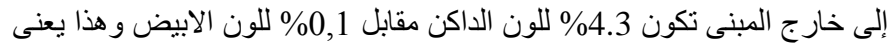

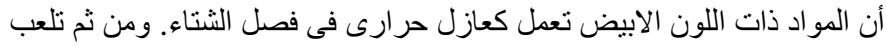

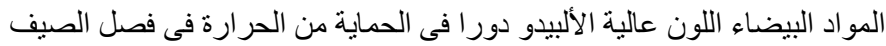

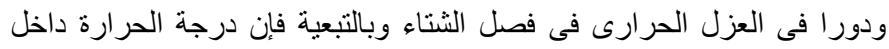

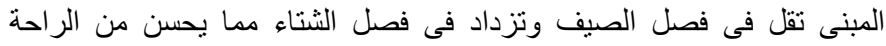
الحرارية و هذا يتفق مع النتائج المنشورة سابقا ويؤكد صحة الحسابات العددية في لئ لئ

البحث.

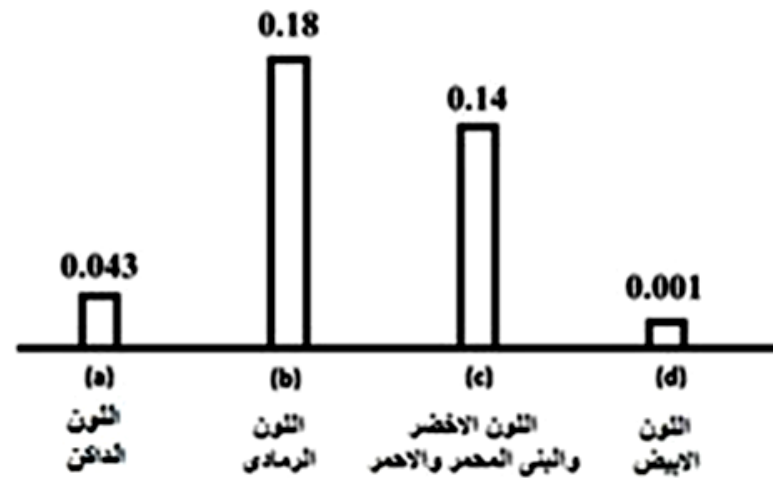

شكل (10):شدة الاشعاع النافذ I داخل المبنى لمو اد ذات ألو ان مختلفة.

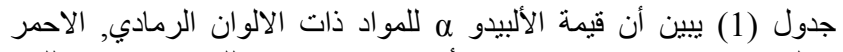

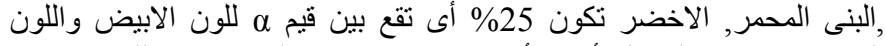

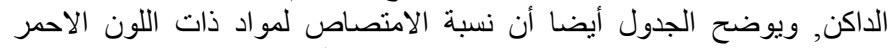

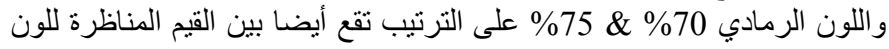
الداكن واللون الابيض و عليه يكون تغير شدة الإشعاع النافذ و المدنص لحائط ذو لُّن

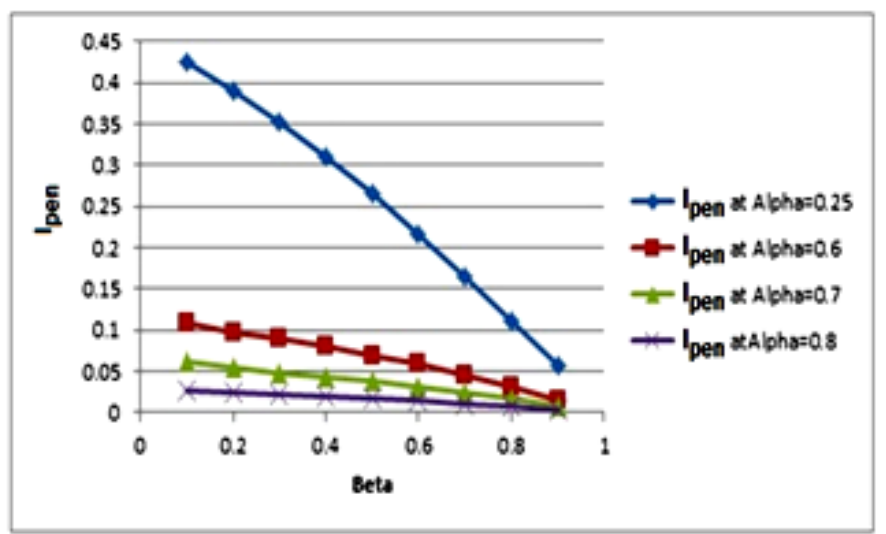

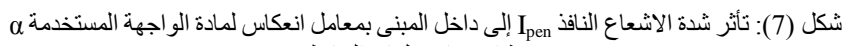
ونسبة امتصاص لمادة الحائط م.

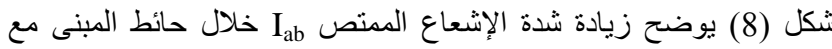

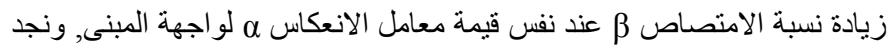

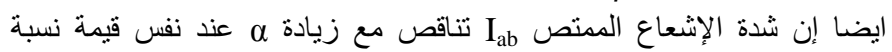

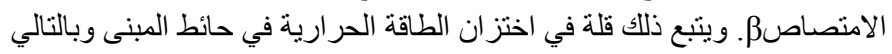

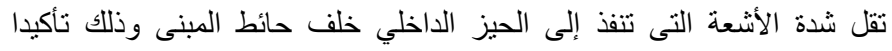

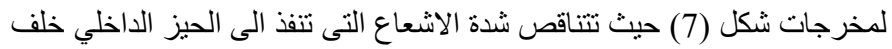

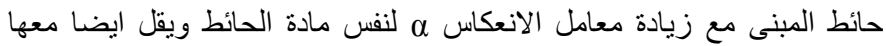

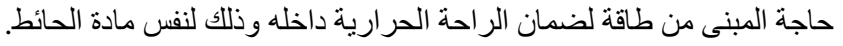

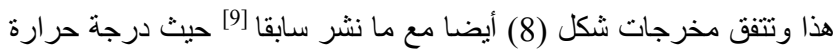

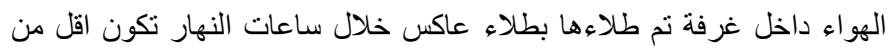

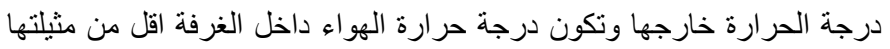

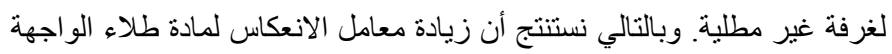

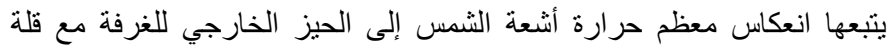

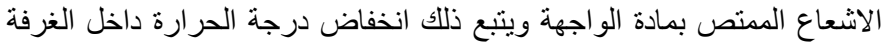
كما تؤكده الحسابات العددية في البحث.

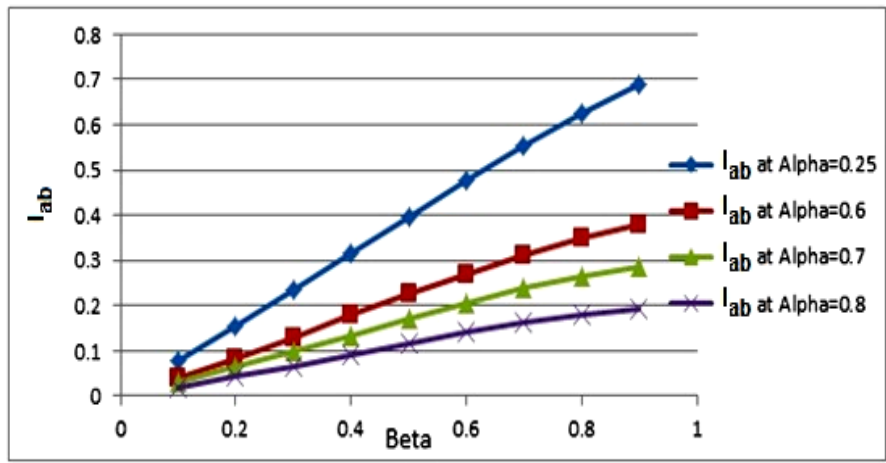

شكل (8): تأثر شدة الاشعاع الممتص Iab فى حائط مبنى بمعامل انعكاس لمادة الو اجهة م ونسبة

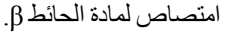

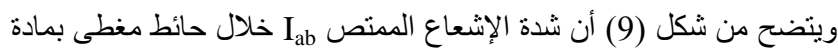

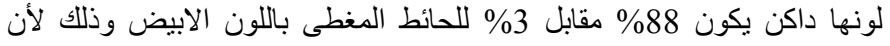

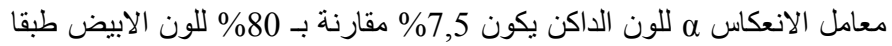

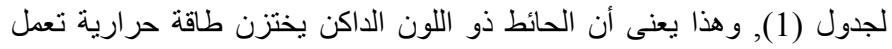

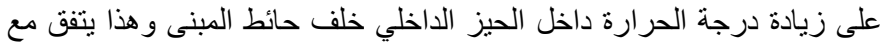

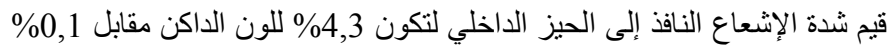
للون الابيض كما يتضح فى شكل (10). 


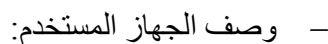
- لإجر اء القياسات الميدانية تم استخدام الجهاز الموضح بثكل رقم (11) وبياناته

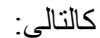

High Tech Computer Corporation الثشركة المصنعة: شركة

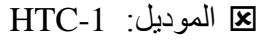

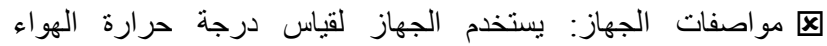
بالسليزيوس (C) وقياس الرطوبة النسبية (\%).

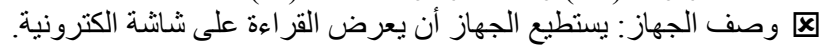

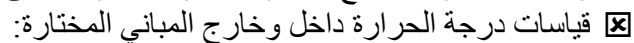

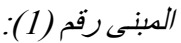

مبنى سكنى بشارع العادلي بحي فريال بمدينة أسيوط واجهته الخارجية

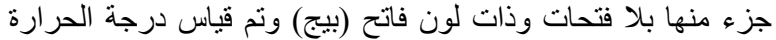

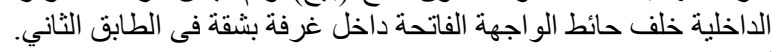

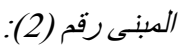
مبنى سكنى بشارع العادلي بحي فريال بمدينة أسيوط واجهته الخارجية

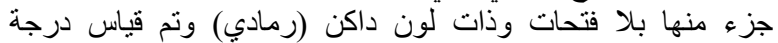

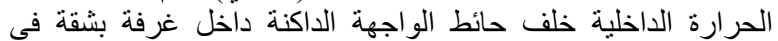

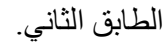

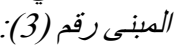
مبنى كلية التربية الرياضية بجامعة اسيوط بمدينة أسيوط واجهته

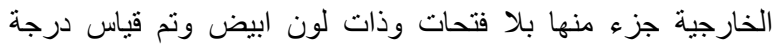

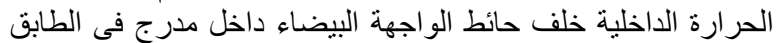

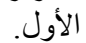

ويوضح شكل (12) الوان واجهات المباني المختارة بينما جدول (2) يبين قيم درجات الحرارة الداخلية والخارجية المقاسة للمباني المختارة.
الالوان الرمادي والاحمر والاخضر فى اعتمادها على معامل الانعكاس م ونسبة

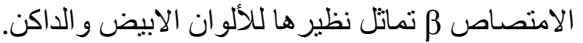
ب- دراسة حالات محلية:

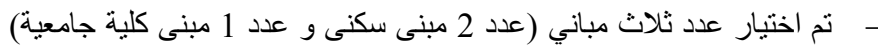
بمدينة أسيوط كدر اسة لحالات محلية, وتم هذا الاختبار طبقا لعدة عو امل: • اختلاف لون الو اجهة الخارجية لهذه المباني.

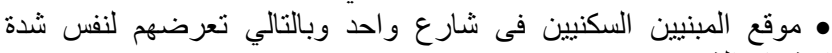
الاشعاع الشمسي. • تعذر وجود مباني أخرى ذات واجهات بدون فتحات لقياس درجة الحرارة

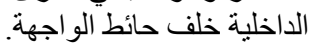
نم قياس درجة الحر ارة الخارجية ودرجة الحة الحر ارة الداخلية الدارة خلف حائط واجهة

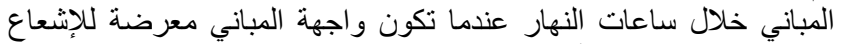

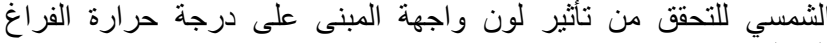
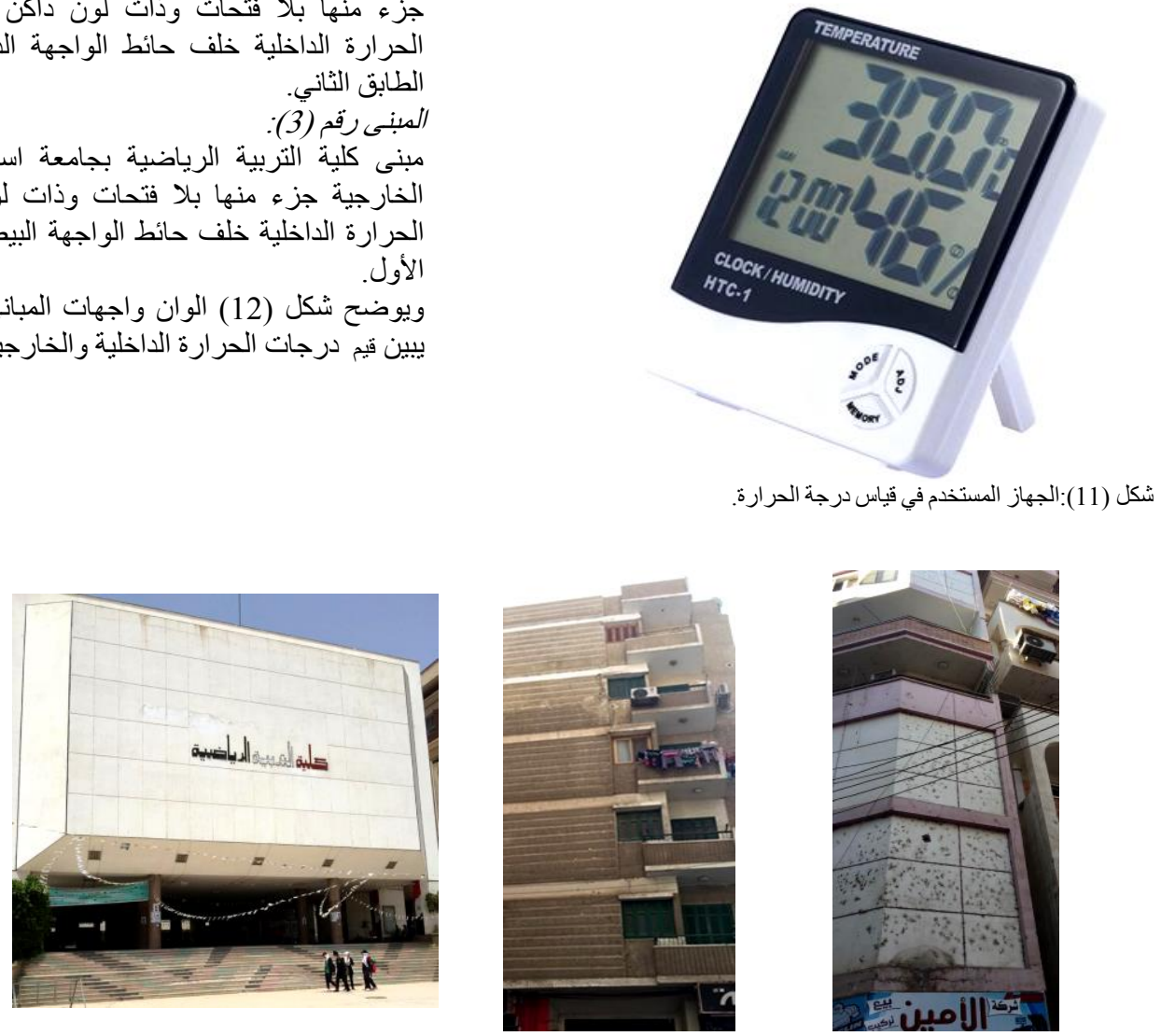

(ج) مبنى كلية التربية الرياضية

(ب) مبنى سكنى رقم (2) - (2) - (ب)

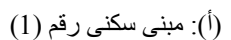

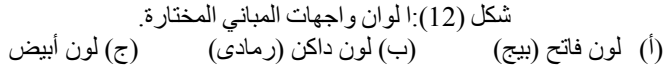

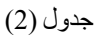

قيم درجات الحرارة الداخلية والخارجية المقاسة للمباني المختارة.

\begin{tabular}{|c|c|c|c|c|c|c|}
\hline \multirow{2}{*}{ فنسبة مرجني الحرارة (\%) } & \multirow{2}{*}{$\begin{array}{c}\text { فرق درجتي الحرارة } \\
\left(T_{O} \text { - TI }\right)\end{array}$} & \multicolumn{2}{|c|}{ درجة الحرارة } & \multirow{2}{*}{ ساعة القياس } & \multirow{2}{*}{ يوم القياس } & \multirow{2}{*}{ رقم المبنى } \\
\hline & & TI الداخلية & To الخارجية & & & \\
\hline $16.7 \%$ & 5.7 & 28.5 & 34.2 & 12 & \multirow{2}{*}{$22 / 04 / 2021$} & \multirow{2}{*}{ المبنى رقم } \\
\hline $19.3 \%$ & 6.2 & 26.0 & 32.2 & 14 & & \\
\hline $11.9 \%$ & 4.1 & 30.3 & 34.4 & 12.30 & \multirow{2}{*}{$22 / 04 / 2021$} & \multirow{2}{*}{ المبنى رقم } \\
\hline $11.2 \%$ & 3.6 & 28.6 & 32.2 & 14.30 & & \\
\hline $42.4 \%$ & 20.2 & 27.4 & 47.6 & 12 & \multirow[t]{2}{*}{$25 / 04 / 2021$} & \multirow[t]{2}{*}{ المبنى رقم } \\
\hline $32.3 \%$ & 14.8 & 31.1 & 45.9 & 14 & & \\
\hline
\end{tabular}




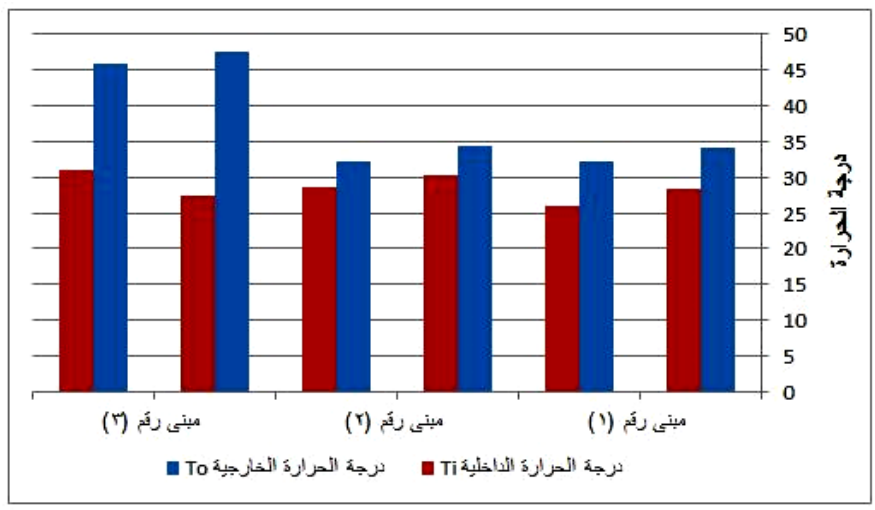

شكل (13): اختلاف كل من درجة الحر ارة الخارجية و الداخلية للثلاثة مباني المختارة.

ج- دراسات مبي/نية:

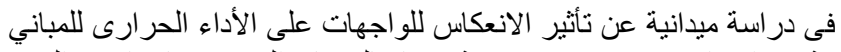

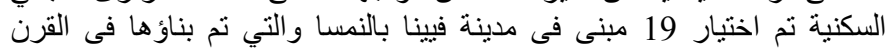

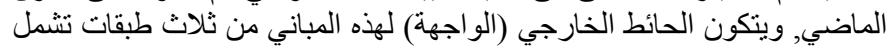

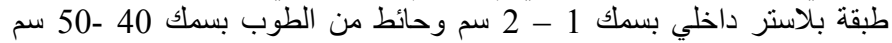

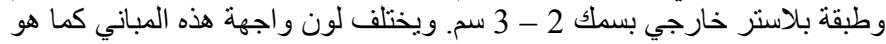

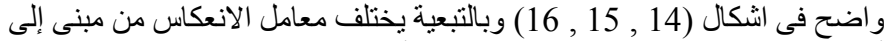

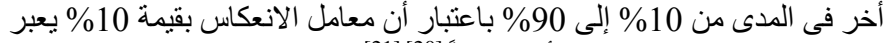
عن و اجهة المبنى ذات اللون الأعلى دكناً [20] [21]
- تحليل نتائج القياسات الموضحة بشكل رقم (12) وجدول (2):

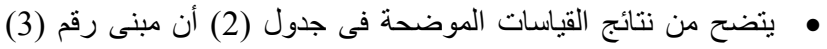

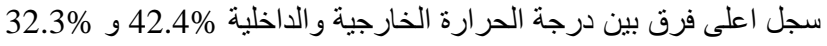

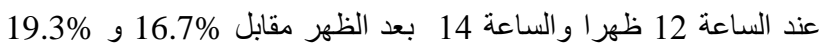
للمبنى رقم (1) و 11.9\% و 11.2\% للمبنى رقم (2).

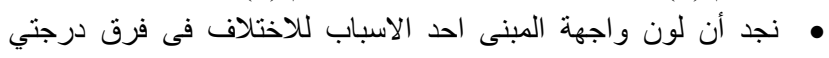

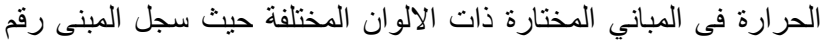
(3) ذو اللون الابيض اعلى فرق فى درجات الحرارة يليه المبنى رقم (1)

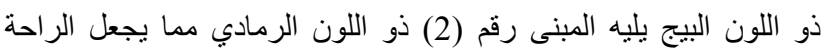

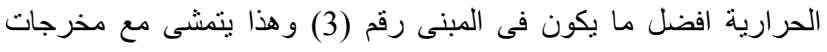

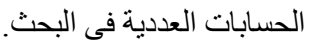

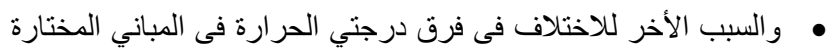

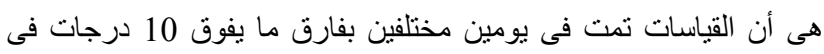
درجة حرارة الجو. • ويوضح شكل (13) اختلاف كل من درجة الحر ارة الخارجية والداخلية

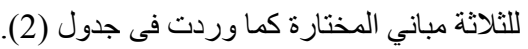
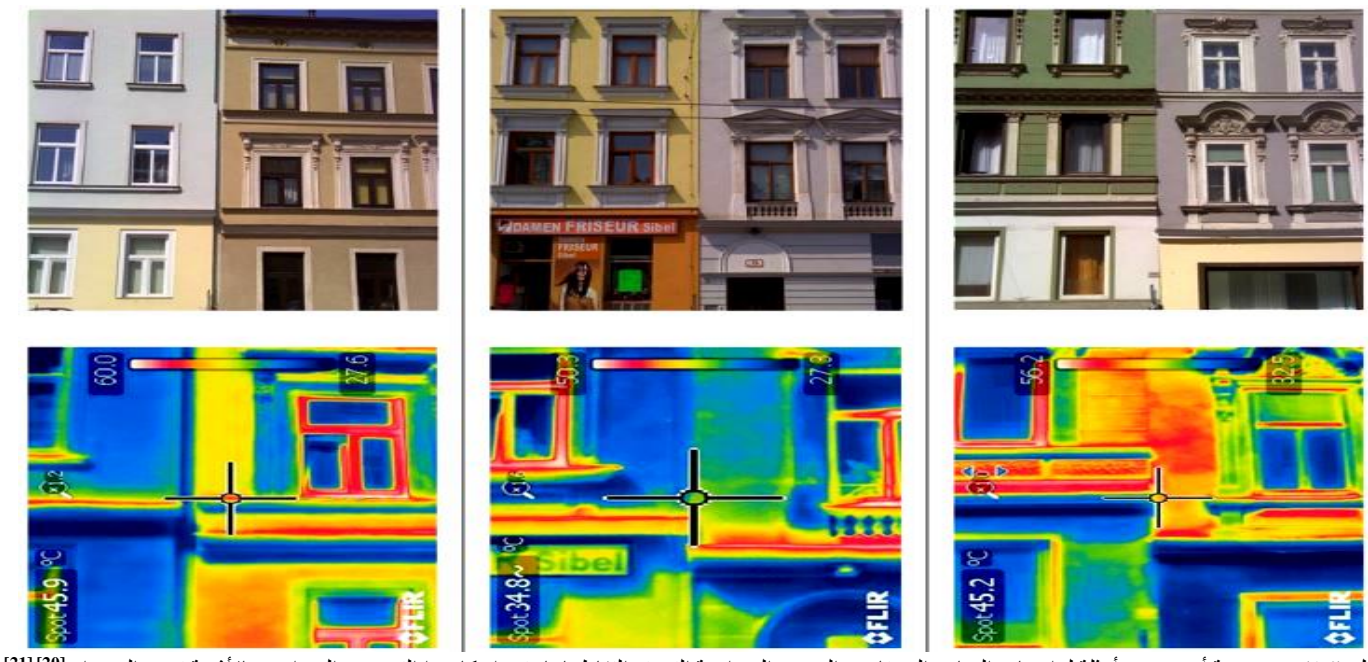

شكل (14): مجمو عة أخرى من أمثلة لواجهات المباني المختارة والصور الحر ارية التى تم التقاطها باستخدام كامير التصوير الحر ارى بالأشعة تحت الحمر اء [21][].
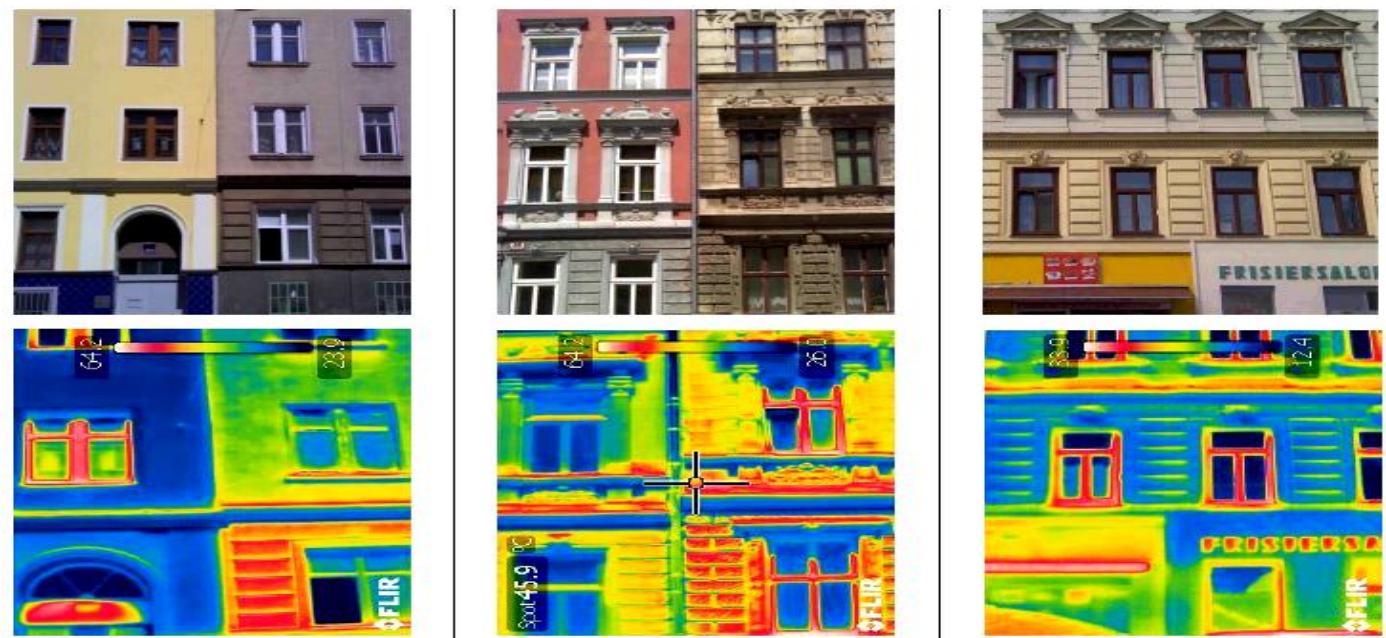

شكل (15): مجموعة أخرى من أمثلة لو اجهات المباني المختارة والصور الحر ارية النى تم التقاطها باستخدام كامير ا التصوير الحر ارى بالأشعة تحت الحمر اء [21] 

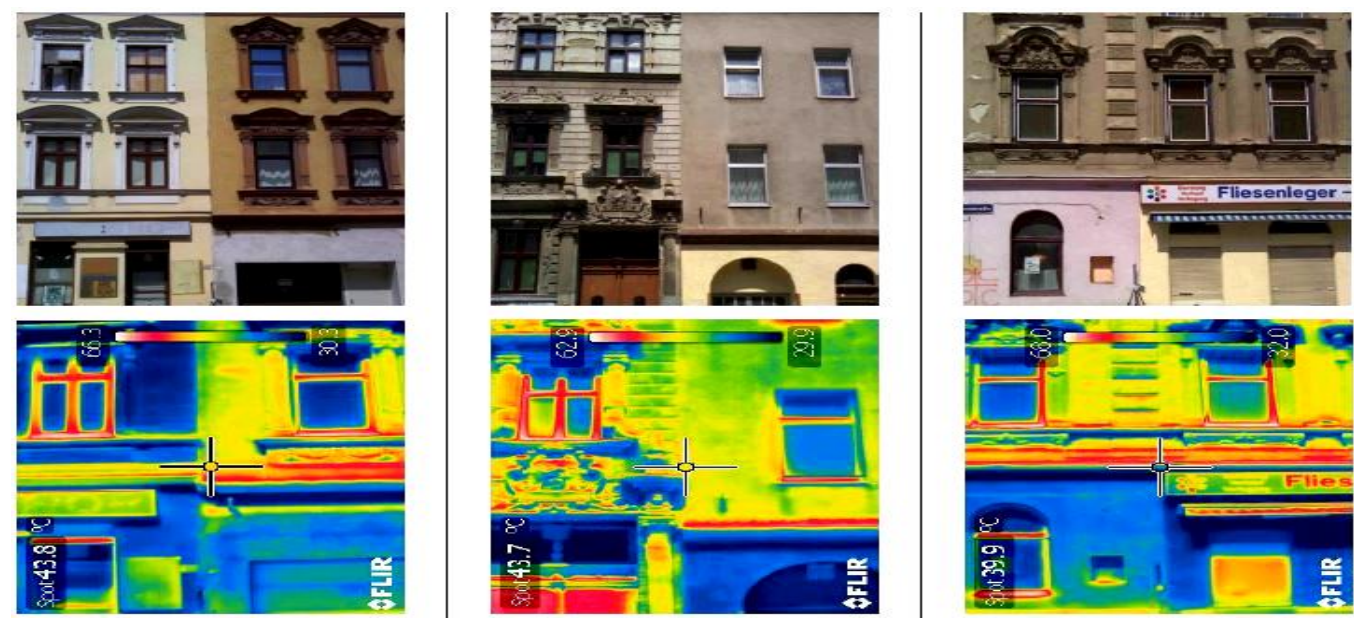

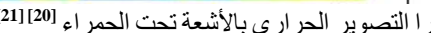

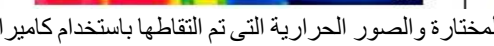

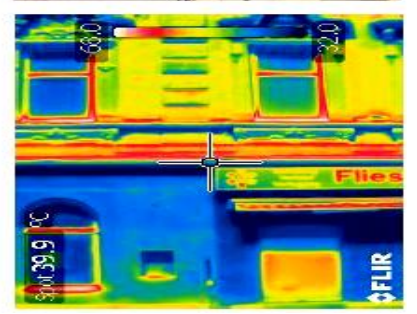

شكل (16): مجمو عة ثالثة من أمثلة لو اجهات المباني المئ

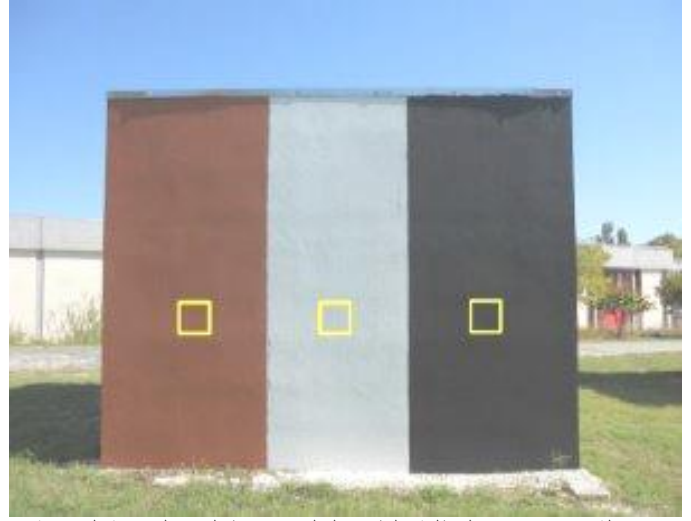

شكل (18): موقع الثلاث مساحات المطلية بالطلاء التقليدي وسط الوان الواجهة الجنوبية لخلية الاختبار المعملية [22]]

وحيث أن البحث يوصى بانعكاس اشعة الثمس من واجهات المباني لزيادة الر احة الحرارية لقاطني المباني إلا أن ذللك له مردود سلبى خارج الهي المبنى بالنسبة

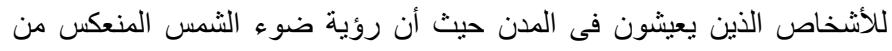

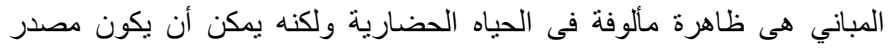

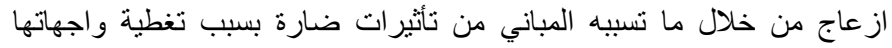
بزجاج عاكس أو ادخال تصاميم منحنية فى الواجهات و المصحوبة بانعكاسات لضوء الثمس [23] ويوجد مثال جيد للنأثيرات الضارة لهذه الانعكاسات وهو مبنى صالة احتفالات ديزني المشهورة Disney Concert Hall فى مدينة لوس انجلوس بكاليفورنيا بأمريكا كما فى شكل (19) حيث أن واجهة المبنى مقعرة الثكل ملئل

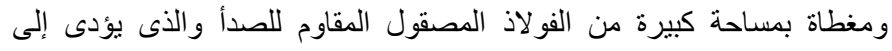

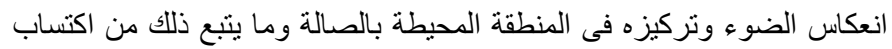

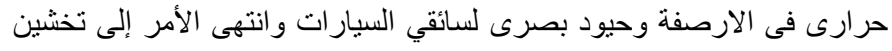

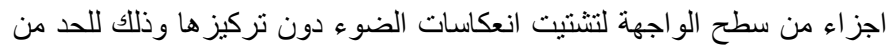
شدة هذه الانعكاسات [23] [24] ويوجد العديد من الأمثلة الحديثة لمباني لها تأثيرات ضارة بسبب الضيب الضوء

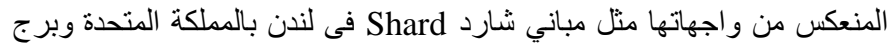

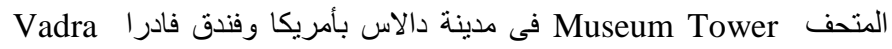

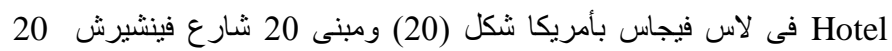
Fenchurch Street الأخيرين أن اشعة الشمس المنعكسة فى مناطق المشاة تسبب حروق عند ارتفاع

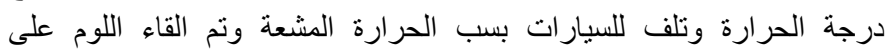

تمت القياسات المعملية لدرجة حر ارة واجهة الـ 19 مبنى وشدة الانارة

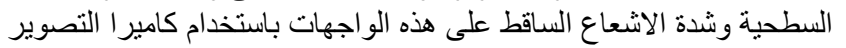
الحرارى بالأشعة تحت الحمر اه اهُ (Infrared thermal imaging camera), ومن ثم امكن حساب معامل الانعكاس لهذه الو اجهات باعتبات الأباره يساوى شدة الانارة

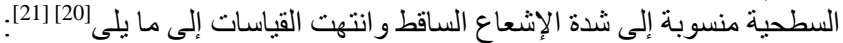

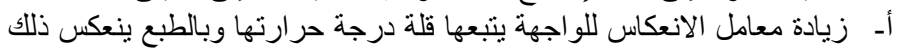

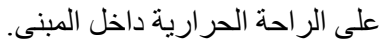

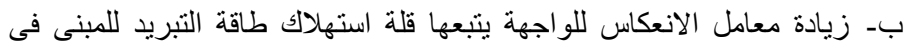
صيف 2012 وصيف 2013 وبالطبع ينعكس ذللك على الراحة الحرارية

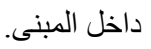
و هذا يتو افق مع ما توصلت إليه الحسابات العددية فى البحث. التئ.

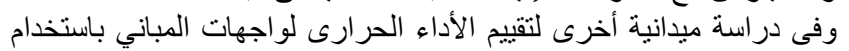

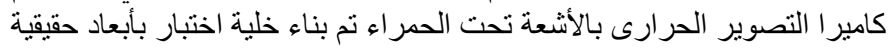

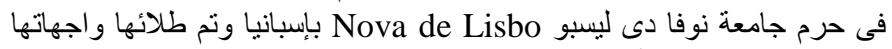

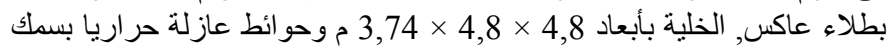

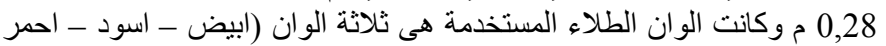

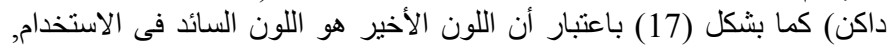

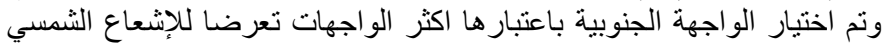

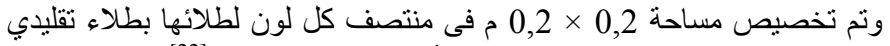

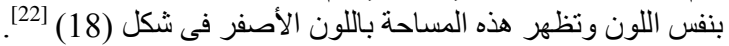

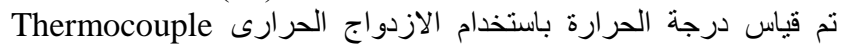

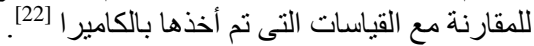

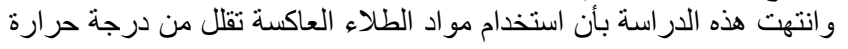

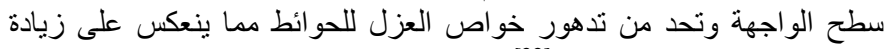
العمر الافتر اضي لهذا الحوائط [22]

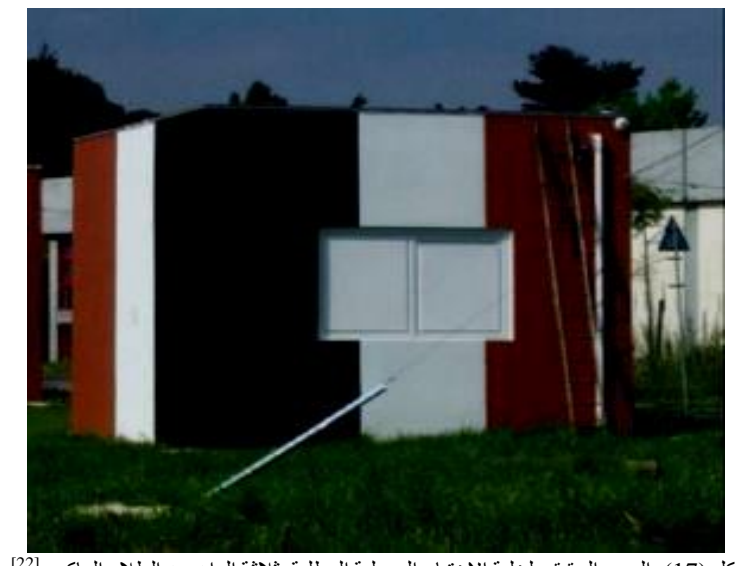

شكل (17): الحجم الحقيقي لخلية الاختبار المعلية المطلية بثلاثة الوان من الطلاء العاكس [22]. 


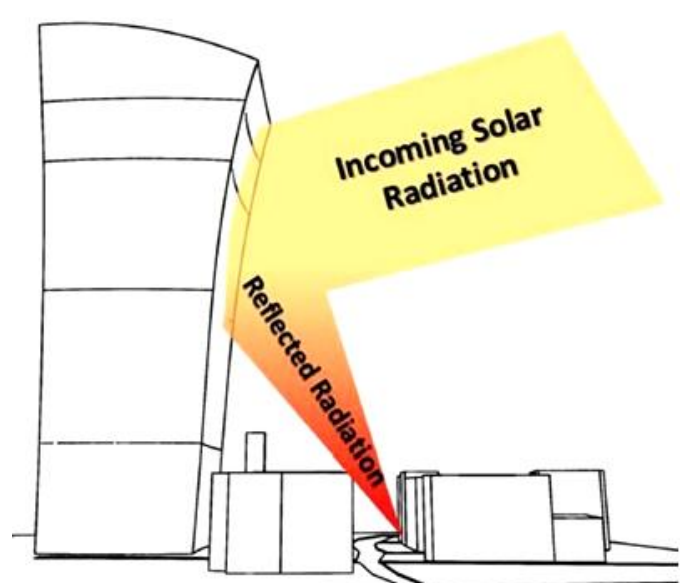

شكل (22): الإشعاع المنعكس من مبنى 20 شارع فينشيرش بلندن بالمملكة المتحدة [25].

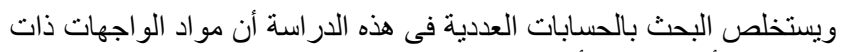

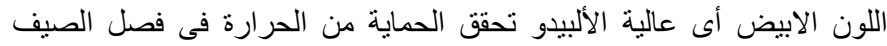

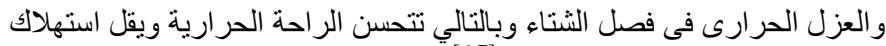

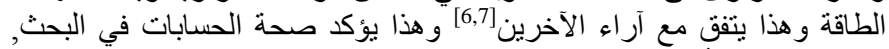

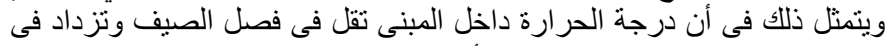
فصل الثتاء مع استخدام مواد عاد دالية الألبيدو.

\section{IX}

من أهم الاستتناجات التى توصل إلبها هذا البحث ما يلى:

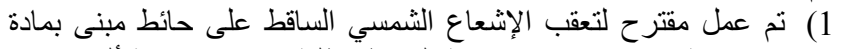

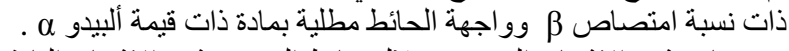

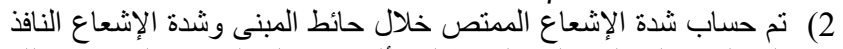

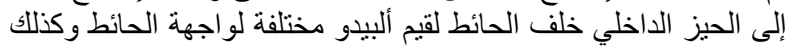
لقيم مختلفة من نسبة الامتصاص لاصلي لمادة الحائط.

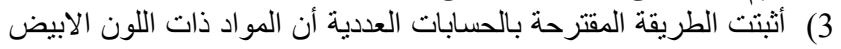

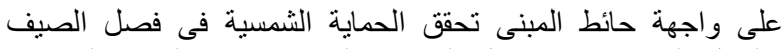

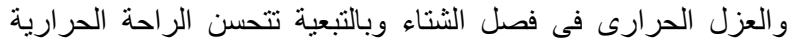
ويقل استهلاك الطاقة. 4) وجد بالحسابات العددية أن درجة الحرارة الطارة داخل المبنى نتيجة استخدام

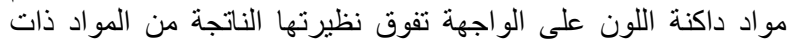
اللون الابيض و هذا يتفق مع آر آلـ الاء الآخرين.

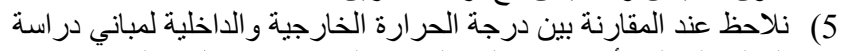

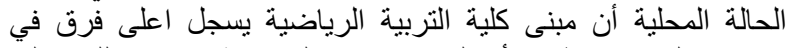

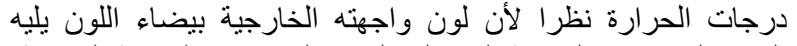

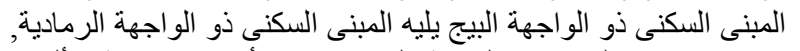

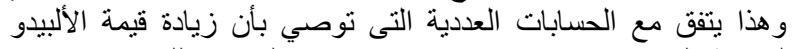

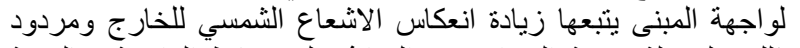

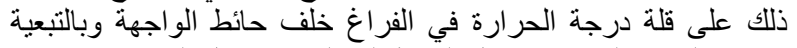
تتحسن الراحة الحرارية داخل الفر اغ الداخلي في فصل الصن الصيف.

\section{X}

من أهم التوصيات التى توصل إليها هذا البحث ما يلى:

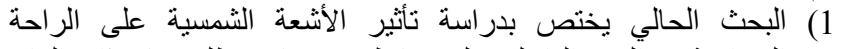

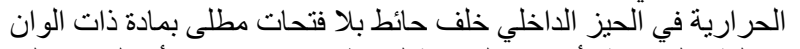

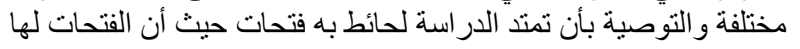

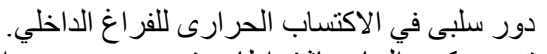

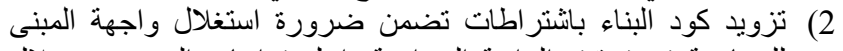

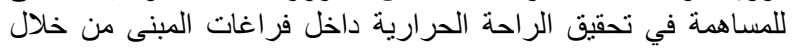

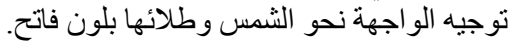

المعماريين لتسببهم فى الاضرار البصرية والحرارية نتيجة هذه الانعكاسات [23]

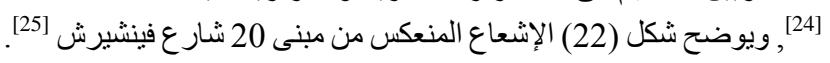

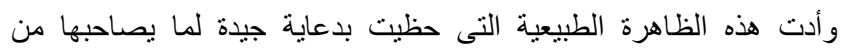

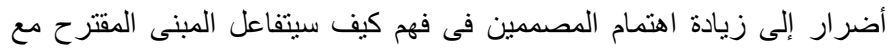
الثمس وكيف تؤثر الانعكاسات من المبنى أثناء تصميمه على التهيف المحيط الخارجي

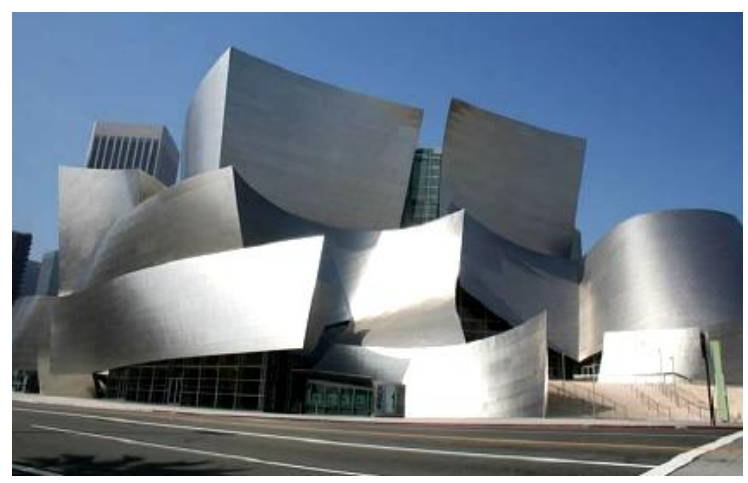

شكل (19): مبنى صالة احتفالات ديزني الثهيرة بمدينة لوس انجلوس بكاليفورنيا [24] [26].

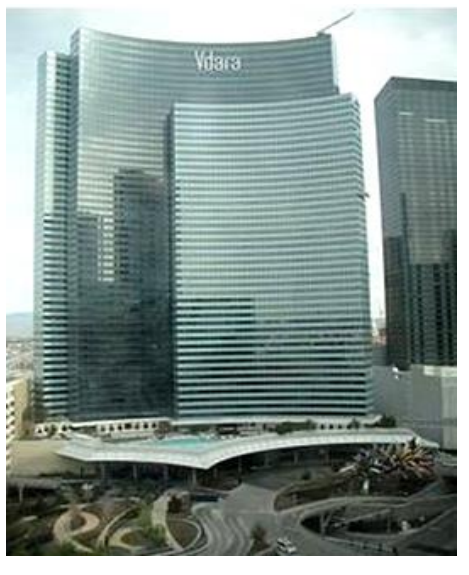

شكل (20): فندق فادر ا في لاس فيجاس بأمريكا [24] [27].

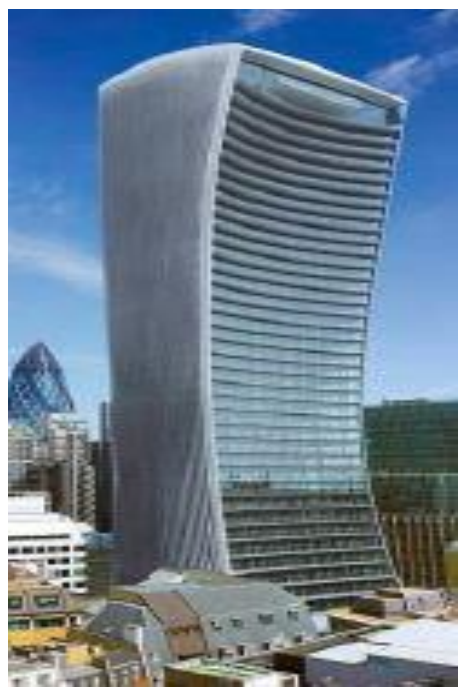

شكل (21): مبنى 20 شارع فينشيرش بلندن بالمملكة المتحدة [24] [28]. 
[18] السقاف, حمحة عبد الله, "عمارة المناطق الحارة ومدى الاستفادة والحماية من الطاقة الثمسية

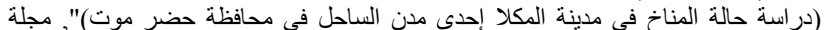

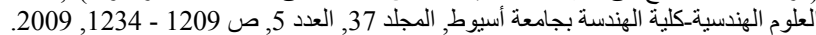

[19] R.A. Serway and J. W. Jewett, Physics for Scientists and Engineers, Chapters 16 and 35, $6^{\text {th }}$ Edition, Thomson Brooks/Cole, USA, 2004.

[20] A. Azarnejad and A. Mahdavi, "Implications of facades visual reflectance for buildings' thermal performance", Journal of Building Physics, Vol. 42, No. 2, pp. 1-17, 2017.

[21] A. Azarnejad, "Impact of building facades' color on building and urban design", Doctor Thesis, Technical University of Vienna, Austria, pp. 2430, 2017.

[22] L. Goncalves, L. Matias and P. Faria, "Thermal performance of cool facades evaluation by infrared thermography", $4^{\text {th }}$ IAHS World Congress on Housing Sustainable Housing: Sustainable Housing Construction, , pp. 1-10, Funchal, Porugal, 16-19 December, 2014.

[23] R. Danks, J. Good and R. Sinclair, "Assessing reflected sunlight from building facades: A literature review and proposed criteria", Building and Environment, Vol. 103, pp. 193-202, 2016.

[24] H. N. Tumbas, "Analyzing the impacts of building form on the environment: A case study in Ankara with a focus on solar reflection", Master Thesis, Middle East Technical University, Turkey, pp. 25-37, 2019

[25] https://www.thoughtco.com/gehry-responds-to-concert-hall-heat178089.

[26] https://en.wikipedia.org/wiki/Vdara.

[27] https://www.loganenergy.com/wp-content/uploads/2015/11/150818-20Fenchurch-Street-GW.pdf

[28] J. Zhu, W. Jahn and G. Rien, "Computer simulation of sunlight consuntration due to façade shape: application to the 2013 death ray at Fenchurch street, London", Journal of Building Performance Simulation", Vol. 12, No. 4, pp. 1-10, 2019.

\section{Title Arabic:}

$$
\text { تأثير الأشعة الثمسية على الر احة الحر ارية فى الحيز الداخلي }
$$

\section{Arabic Abstract:}

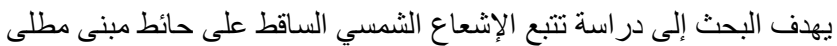

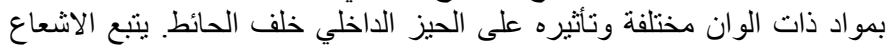

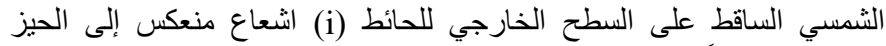

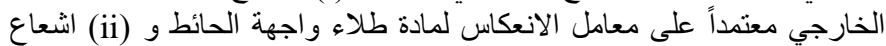

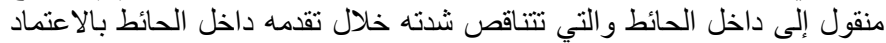

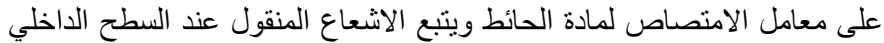

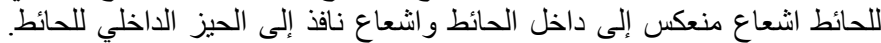

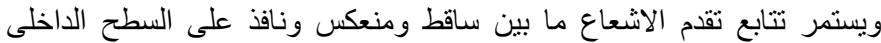

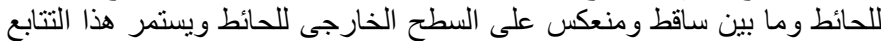

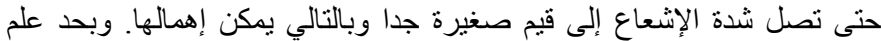

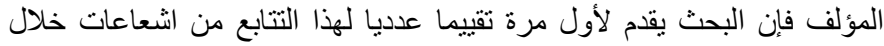

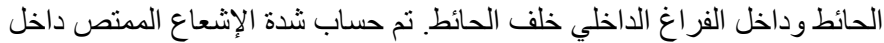

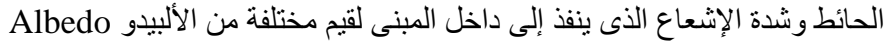

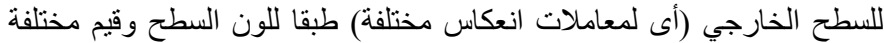

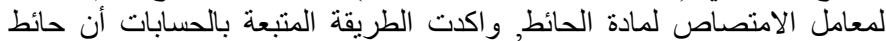

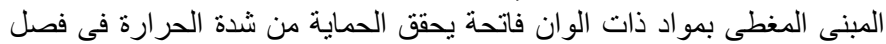

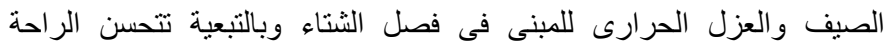

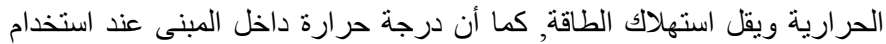

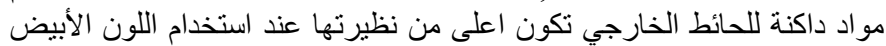

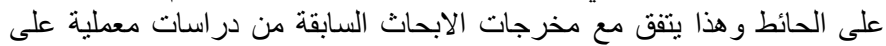

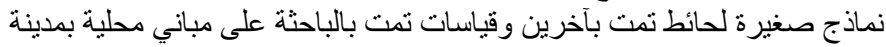

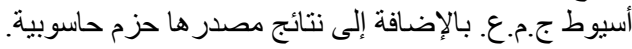

إلزام المهندسين بتطبيق كود البناء بهدف تحقيق الراحة الحرارية في

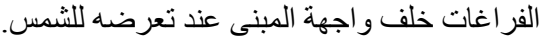
4) حث المصممين على استخدام المواد العاكسة على واجلى واجهات المباني التى التى تكتسب حر ارة عالية صيفا.

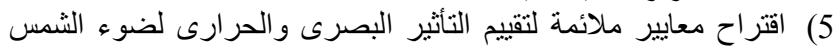

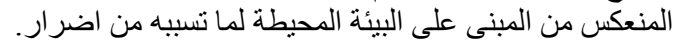

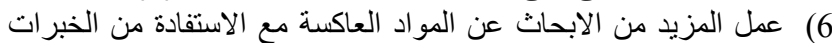

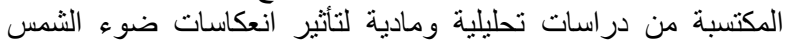
لمباني قائمة.

\section{شكر}

تشكر الباحثة دكتور مهندس/ أيمن عبد الرحيم عمرو مدير عام الصيانة بشركة الصنة

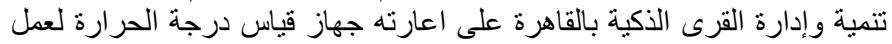
القياسات التى وردت في البحث.

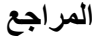

[1] القطينى, فادى - مزاحم, زين الدين - ديون, طالب, "التكوين الخارجي للمبنى وتأثيره على التى الراحة الحرارية للمستعطلين", مجلة جامعة البعث, المجد 38, العدد 3, ص صل 143 - 179, 2016

[2]R. Siegel and J. Howell, "Thermal Radiation Heat Transfer", Taylor \& Francis publisher, 4th Edition, 2001.

[3]C. Strobel, M. Abadie and N. Mendes, "Absorption of solar radiation in thick and multilayered glazing", Vol. 10, pp. 238-244, Beijing, China, September 3-6,

2007 , https://www.aivc.org/sites/default/files/p583_final.pdf

[4]N. K. Bansal, S. N. Garg and S. Kothari, "Effect of exterior surface color on the thermal performance of buildings", Building and Environment, Vol. 27, No. 1, pp. 31-37, 1992.

[5]E. Cheng, E. Ng and B. Givoni, "Effect of envelope colour and thermal mass on indoor temperatures in hot humid climate", Solar Energy, Vol. 78, No. 4, pp. 528-534, April 2005..

[6]B. Al-hafiz, M. Musy and T. Hasan, "A study of the impact of changes in the materials reflection coefficient for achieving sustainable urban design", Procedia Environmental Sciences, Vol. 38, pp. 562-570, 2017.

[7]B. Yu, Z. Chen, P. Chang and Y. Yang, "Study of the influence of albedo on building heat environment in a year-round", Energy and Buildings, Vol. 50, No. 5, pp. 945-951, 2008.

[8]Q. Mansouri, R. Belarbi and F. Bourbia, "Albedo effect of external surfaces on the energy loads and thermal comfort in buildings", Energy Procedia, Vol. 139, pp. 571-577, December, 2017.

[9]G. Xing-gue, W. Jia, W. Yuc, A. Yu-qiang and L. Xiang-wei, "Experimental Study of the thermal performance of a new type of building reflective coating in hot summer and cold winter zone of China", Procedia Engineering, Vol. 205, pp. 603-608, October, 2017.

[10] H. Taha, H. Akbari, H. Rosenfeld, et al., "Residential cooling loads and the urban heat island: the effects of albedo", Building and Environment, Vol. 23, No. 4, pp. 271-283, 1988.

[11] H. Akbari and H. Taha,, "The impact of trees and white surface on residential heating and cooling energy use in four Canadian cities", Energy, Vol. 17, No. 2, pp. 141-149, 1992.

[12] K. Tarabieh, A. Aboulmagd, "Thermal Performance Evaluation of Common Exterior Residential Wall Types in Egypt", Buildings, Vol. 9, https://doi:10.3390/buildings9040095, April, 2019.

[13] https://ar.wikipedia.org/wiki/\%D9\%82\%D8\%B5\%D8\%A7\%D8\%B1\% D8\%A9

[14] العيسوى, تحمح عبد الفتاح أحمد, "تأثيّر تصميم الغلاف الخارجي للمبنى على الاكتساب الحرارى

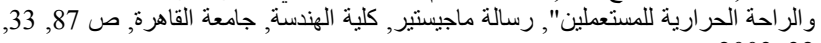
. 2003,32

[15] الوكيل, شفق العوضى - سراج, محمد عبد الله, "المناخ وعمارة المناطق الحارة", مكتبة دار

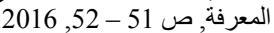

[16] عباس, آرام عبد العزيز السنى, "أثر التصميم المناخي على تحقيق الراحة الحرارية

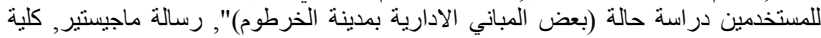

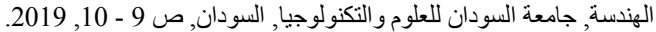

[17] http://repository.sustech.edu/bitstream/handle/123456789/15705/\%D8\% A7\%D9\%84\%D8\%A8\%D8\%AD\%D8\%AB.pdf?sequence=2\&isAllowe $\mathrm{d}=\mathrm{y}$ 